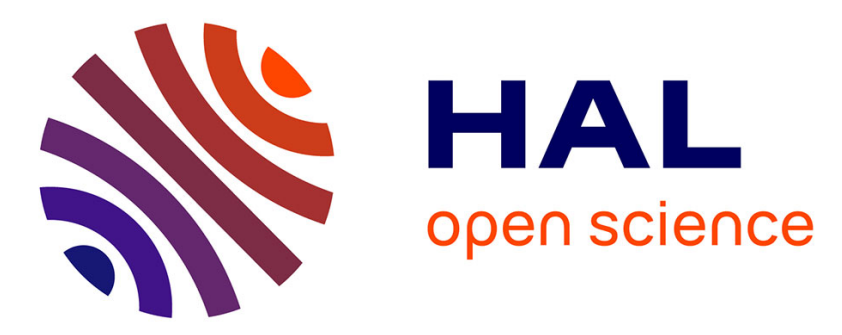

\title{
Flow of yield stress and Carreau fluids through rough-walled rock fractures: Prediction and experiments
}

\author{
Antonio Rodríguez de Castro, Giovanni Radilla
}

\section{To cite this version:}

Antonio Rodríguez de Castro, Giovanni Radilla. Flow of yield stress and Carreau fluids through rough-walled rock fractures: Prediction and experiments. Water Resources Research, 2017, 53 (7), pp.6197-6217. 10.1002/2017WR020520 . hal-02320018

\section{HAL Id: hal-02320018 https://hal.science/hal-02320018}

Submitted on 18 Oct 2019

HAL is a multi-disciplinary open access archive for the deposit and dissemination of scientific research documents, whether they are published or not. The documents may come from teaching and research institutions in France or abroad, or from public or private research centers.
L'archive ouverte pluridisciplinaire HAL, est destinée au dépôt et à la diffusion de documents scientifiques de niveau recherche, publiés ou non, émanant des établissements d'enseignement et de recherche français ou étrangers, des laboratoires publics ou privés. 
1 Flow of Yield Stress and Carreau fluids through Rough-Walled Rock Fractures:

2 prediction and experiments

3 Antonio Rodríguez de Castro* $(1,2)$, Giovanni Radilla $(1,3)$

4

5

(1) Arts et Métiers ParisTech, 51006 Châlons-en-Champagne, France

6

(2) Laboratoire MSMP - EA7350, Rue Saint Dominique, 51006 Châlons-en-Champagne,

7 France

8

(3) LEMTA, UMR 7563, CNRS, 54518 Vandœuvre-lès-Nancy, France

9

10

11

$12 *$ Corresponding author

13 Dr. Antonio Rodríguez de Castro

14 Arts et Métiers ParisTech

15 Rue Saint-Dominique

1651006 Châlons-en-Champagne

17 France

18 Tel: +33326699173 
19 Email: antonio.rodriguezdecastro@ensam.eu

20 


\section{Abstract}

Many natural phenomena in geophysics and hydrogeology involve the flow of non-

Newtonian fluids through natural rough-walled fractures. Therefore, there is considerable interest in predicting the pressure drop generated by complex flow in these media under a given set of boundary conditions. However, this task is markedly more challenging than the Newtonian case given the coupling of geometrical and rheological parameters in the flow law. The main contribution of this paper is to propose a simple method to predict the flow of commonly used Carreau and yield stress fluids through fractures. To do so, an expression relating the "in-situ" shear viscosity of the fluid to the bulk shear-viscosity parameters is obtained. Then, this "in-situ" viscosity is entered in the macroscopic laws to predict the flow rate-pressure gradient relations. Experiments with yield stress and Carreau fluids in two replicas of natural fractures covering a wide range of injection flow rates are presented and compared to the predictions of the proposed method. Our results show that the use of a constant shift parameter to relate "in-situ" and bulk shear viscosity is no longer valid in the presence of a yield stress or a plateau viscosity. Consequently, properly representing the dependence of the shift parameter on the flow rate is crucial to obtain accurate predictions. The proposed method predicts the pressure drop in a rough-walled fracture at a given injection flow rate by only using the shear rheology of the fluid, the hydraulic aperture of the fracture and the inertial coefficients as inputs. 


\section{Introduction}

The flow of complex fluids through rough-walled rock fractures is involved in many economically important industrial applications, such as soil remediation, hydrogeology or Enhanced Oil Recovery (EOR) [Radilla et al., 2013; Tosco et al., 2013; Coussot, 2014]. Numerous complex fluids are shear-thinning, showing a decrease in shear viscosity as the applied shear rate is increased. Shear-thinning fluids are extensively used in petroleum engineering and soil remediation to improve the microscopic sweep of the reservoir through stabilization of the injection front [Lake, 1989; Silva et al., 2012; Wever et al., 2011]. For instance, shear-thinning drilling fluids containing the biopolymer xanthan [Zhong et al., 2008; Truex et al., 2015] and other polymers such as polyacrylamide [Ball and Pitts, 1984], carboxymethylcellulose [Zhang et al., 2016] and guar gum [Hernández-Espriú et al., 2013] are widely used in EOR.

In some cases, fluids with shear-rate dependent viscosity also present a yield stress, i.e. a threshold value in terms of shear stress below which they do not flow. Many complex fluids used in industrial applications exhibit yield stress behaviour, e.g. polymer solutions, waxy crude oils, volcanic lavas, emulsions, colloid suspensions, foams, etc. [Coussot, 2005; Dimitriou and McKinley, 2015; Roustaei et al., 2016; Talon et al., 2014 ; Lavrov, 2013; Coussot, 2014].Common examples of yield stress shear-thinning fluids are the slurries or cement grouts injected to reinforce soils, the heavy oils or the drilling fluids injected into rocks for the reinforcement of wells [Lavrov, 2013; Coussot, 2014]. Indeed, drilling fluids are often designed so as to have a yield stress in order to prevent cutting from settling when circulation stops [Lavrov, 2013]. Also, a number of fracturing fluids used in hydraulic 
fracturing exhibit a yield stress designed to enhance proppant transport [Talon et al., 2014; Roustaei et al., 2016] and present shear-thinning behaviour [Lavrov, 2015; Perkowska et al., 2016].

For these reasons, the flow of shear-thinning fluids in porous media, and in particular that of yield stress fluids, has become a field of great research interest [Chevalier et al., 2013; Chevalier et al., 2014; Coussot, 2014; Talon et al., 2014; Rodríguez de Castro et al., 2016]. However, although recent advances have been made [Chevalier et al., 2013; Chevalier et al., 2014], obtaining a macroscopic law to predict pressure drop as a function of flow rate has proved to be a stumbling-block. Also, despite its broad interest, a serious lack of experimental works involving the flow of yield stress fluids was reported by Lavrov [2013] and Coussot [2014].

Inspired by the growing scope of industrial applications in which shear-thinning and yield stress fluids are injected through rough-walled fractures, the objective of this work is to present a simple method to predict the pressure losses generated during single-phase flow. The accuracy of the resulting predictions is then evaluated through comparison with experimental data. To do so, a series of flow experiments with concentrated aqueous solutions of xanthan biopolymer presenting a yield stress were carried out by measuring the pressure drop as a function of the injection flow rate during the flow through two replicas of rough-walled natural fractures (granite and Vosges sandstone). Furthermore, previously presented experimental data involving the flow of shear-thinning with no yield stress are also compared with the predictions obtained with the proposed method. 
90

$$
\nabla \mathrm{P}=\frac{\mu}{\mathrm{K}} \frac{\mathrm{Q}}{\mathrm{A}}=\frac{\mu}{\mathrm{K}} \mathrm{u}
$$

$94 \nabla \mathrm{P}=\frac{\Delta \mathrm{P}}{\mathrm{L}}$ being the pressure gradient, $\Delta \mathrm{P}$ the absolute value of the pressure drop over a

The single-phase flow of incompressible Newtonian fluids through porous media is governed by Darcy's law [Darcy, 1856]. In the case of one-directional steady flow through a horizontal porous media, this law is written as:

distance L, Q the volumetric flow rate, A the cross-sectional area, $\mathrm{u}=\mathrm{Q} / \mathrm{A}$ the average velocity, $\mu$ the viscosity of the injected fluid, and $\mathrm{K}$ the intrinsic permeability. This model is restricted to creeping flow in which inertial forces are negligible compared to viscous forces [Schneebeli, 1955; Hubbert, 1956; Scheidegger, 1960; Chauveteau and Thirriot, 1967]. Nonlinearity of fluid flow stems from inertial pressure losses generated by the repeated accelerations and decelerations due to rapid changes in flow velocity and direction along the flow path. Both theoretical and empirical models taking into account the extra pressure losses due to inertial effects were presented in the literature [Miskimins et al., 2005]. The results of these studies confirm the existence of a strong inertial regime and a weak inertial regime. The nonlinear behaviors associated to those regimes can be described respectively by a quadratic and a cubic function of the average velocity. Forchheimer's empirical law [Forchheimer, 1901] is commonly used to model the strong inertial regime through addition of a quadratic flow rate term to Darcy's law: 
$\nabla \mathrm{P}=\frac{\mu}{\mathrm{K}} \mathrm{u}+\beta \rho \mathrm{u}^{2}$

110 where $\rho$ is the fluid density and $\beta$ is the inertial coefficient. Forchheimer's law has been

111 experimentally validated [Dullien and Azzam, 1973; Geertsma, 1974; MacDonald et al.,

112 1979; Rasoloarijaona and Auriault, 1994; Javadi et al., 2014; Rodríguez de Castro and

113 Radilla, 2016a] and has found some theoretical justifications [Cvetkovic, 1986; Giorgi, 1997;

114 Chen et al., 2001]. In the case of the weak inertial regime, which occurs at moderate values

115 of the Reynolds number, deviations from the linear relationship between flow rate and

116 pressure loss were shown to follow a cubic function of the mean velocity in the porous media

117 [Mei and Auriault, 1991; Firdaouss et al., 1997; Fourar et al., 2004; Rocha and Cruz, 2010].

118

$\nabla P=\frac{\mu}{K} u+\frac{d \rho^{2}}{\mu} u^{3}$

where $\mathrm{d}$ is a dimensionless inertial coefficient. Reynolds number can be specifically defined for weak inertia cubic law as [Radilla et al., 2013; Rodríguez de Castro and Radilla, 2016a].

$\operatorname{Re}_{c}=\sqrt{\mathrm{Kd}} \frac{\rho}{\mu} \frac{\mathrm{Q}}{\mathrm{A}}$

123 Cubic law was obtained from numerical simulations in a $2 \mathrm{D}$ periodic porous medium 124 [Barrère et al., 1990; Fidarous and Guermond, 1995; Amaral Souto and Moyne, 1997] and also by using the homogenization technique for isotropic homogeneous porous media [Mei 
and Auriault, 1991; Wodie and Levy, 1991]. This law was shown to be in agreement with experimental data [Firdaous et al., 1997; Rodríguez de Castro and Radilla, 2016a].

Using the asymptotic expansions method in a thin cylindrical channel with oscillating walls and averaging over the channel diameter, Buès et al. [2004] and Panfilov and Fouar [2006] presented a macroscopic flow equation which proved to be in good agreement with numerical simulations in rectangular and cylindrical fractures at high flow rates. This flow equation was expressed in the form of a full cubic law:

$\nabla P=\frac{\mu}{K} u+\beta \rho u^{2}+\frac{d \rho^{2}}{\mu} u^{3}$

where $\beta$ and $d$ are the inertial coefficients which may be positive or negative, depending on the channel geometry. $\beta$ and $d$ were shown to be independent of the shear rheology of the injected fluid in previous numerical [Firdaouss et al., 1997; Yadzchi and Luding, 2012; Tosco et al., 2013] and experimental works [Rodríguez de Castro and Radilla, 2016a; Rodríguez de Castro and Radilla, 2016b]. In this full cubic law, the quadratic term describes the pure inertia effect caused by an irreversible loss of kinetic energy due to flow acceleration and the cubic term corresponds to a cross viscous-inertia effect caused by the streamline deformation due to inertia forces. This macroscopic flow equation is valid not only in the Darcian flow regime but also, to some limited extent, for the non-Darcian flow regimes. $\beta$ and $\mathrm{d}$ can be obtained either through fitting to experimental data [Dukhan et al., 2014; Rodríguez de Castro and Radilla, 2016a, 2016b] or through theoretical predictions obtained from porosity, permeability and roughness of the porous medium [Cornell and Katz, 1953; Geertsma, 1974; Neasham, 1977; Noman and Archer, 1987; López, 2004, Agnaou et al., 2016]. 
149 Analogously to the case of cubic law, Reynolds number can be defined for full cubic law as

151

$$
\operatorname{Re}_{\mathrm{fc}}=\frac{\mathrm{K} \beta \rho \mathrm{u}}{\mu}
$$

Previous experimental works demonstrated that Darcy's law fails to predict pressure drops in fractures when inertial effects are relevant [Zimmerman et al., 2004; Radilla et al., 2013; Javadi et al., 2014; Rodríguez de Castro and Radilla, 2016a, 2016b]. Zimmerman et al. [2004] presented experimental data on non-creeping flow through a rock fracture, showing good agreement with Forchheimer's model. The same authors also proved, via numerical solution of the Navier-Stokes equations, the existence of the weak inertia regime for moderate values of Reynolds numbers. Radilla et al. [2013] modelled single-phase flow experiments by means of the full cubic law and presented an elegant method to compare fractures in terms of hydraulic behaviour versus flow regime using the intrinsic hydrodynamic parameters. Besides, a geometrical model for non-linear fluid flow through rough fractures was proposed and evaluated through numerical simulations by Javadi et al. [2010]. More recently, Roustaei et al. [2016] numerically investigated the 2D-flow of a yield stress fluid along an uneven fracture, showing that important Darcy-type flow law lead to important errors in the case of short fractures due to self-selection of the flowing region and the existence of fouling layers of unyielded fluid. 


$$
\mathrm{K}=\frac{\mathrm{h}^{2}}{12}
$$

$$
\mathrm{A}=\mathrm{hw}
$$

175 The aperture distribution of rough-walled rock fractures always presents a strong heterogeneity, due to the wide range of aperture sizes and the significant number of contact points [Witherspoon et al., 1980; Xiong et al., 2011; Javidi et al., 2014; Wang et al., 2016].

178 The hydraulic behaviour through a fracture is known to be heavily dependent upon the apertures distribution [Isakov et al., 2001; Javidi et al., 2014; Wang et al., 2016]. This is explained by the tendency of the fluid to flow through the paths with the largest apertures. Moreover, within a given path, the hydraulic behaviour of fracture would be controlled by the small apertures and constrictions [Tsang and Tsang, 1987; Neuzil and Tracy, 1981].

Several attempts have been made to obtain a macroscopic law linking the injection flow rate to the resulting pressure drop during the flow of yield stress fluids in porous media [Pascal, 1983; Al-Fariss and Pinder, 1987; Chase and Dachavijit, 2005; Coussot, 2014]. A major drawback of most available expressions is the existence of experimentally adjustable 
parameters with no clear physical meaning as inputs, which impedes direct computational predictions. As an alternative, some pore-network approaches have also been proposed [Chen et al., 2005; Sochi and Blunt, 2008]. The main advantage of pore-network models is that they provide a reasonably realistic description of the reality in which the number of flow paths through the porous media increases with the applied pressure gradient. Nonetheless, these effects need experimental validation. Also, pore-network methods do not lead to analytical $\nabla \mathrm{P}-\mathrm{u}$ expressions, which is particularly aggravating in applications involving a wide range of injection flow rates. An important difference between Newtonian and complex fluids is the coupling of geometrical and rheological parameters in the flow law [Roustaei et al., 2016]. Indeed, a non-toxic method of porosimetry has been proposed, which is based on the injection of yield stress fluids through porous media and takes advantage of the mentioned coupling [Ambari et al., 1990; Malvault, 2013; Oukhlef et al. 2014; Rodríguez de Castro, 2014; Rodríguez de Castro et al., 2014; Rodríguez de Castro et al., 2016a].

The literature survey conducted by Lavrov [2013] revealed the severe lack of research on fracture flow of non-Newtonian fluids, especially regarding yield-stress fluids. The earlier works of Di Federico [Di Federico, 1997; Di Federico, 1998; Di Federico, 2001] mainly focused on defining and estimating the equivalent aperture for flow of a non-Newtonian fluid in a variable aperture fracture, without experimental validation. Also, Silliman [1989] provided different aperture estimates for variable aperture fractures. Only a few experimental works exist for the flow of yield stress fluids in porous media [Al-Fariss and Pinder, 1987; Chase and Dachavijit, 2005; Chevalier et al., 2013; Chevalier et al., 2014; Rodríguez de Castro, 2016a], and the ranges of variation of $\mathrm{u}$ are usually narrow. These experimental works show that the relationship between $\nabla \mathrm{P}$ and $\mathrm{u}$ is of the same form as the constitutive equation of the fluid, i.e. $\nabla \mathrm{P}=\nabla \mathrm{P}_{0}+\mathrm{Cu}^{\mathrm{n}}$ with $\nabla \mathrm{P}_{0}$ being the critical pressure gradient below 
which no flow occurs, $\mathrm{n}$ being the flow index of the fluid and $\mathrm{C}$ being a parameter that depends on the porous medium and the boundary conditions. Chevalier et al. [2014] used an NMR imaging technique to show that the velocity density distribution of a yield stress fluid flowing through a packed bed was similar to that of a Newtonian fluid due to the minor role played by the constitutive equation of the fluid in rapidly varying pore geometry. On the basis of these results, the latter authors propose explicit (but complex) expressions with physical meaning to calculate $\nabla \mathrm{P}_{0}$ and C. Concerning multiphase flow, Boronin et al., [2015] developed a model for the displacement of yield-stress fluids in a vertical Hele-Shaw cell and used it to investigate the joint effect of viscous fingering, yielding and gravitational slumping, showing that unyielded fluid zones develop as a result of viscous fingering generated when a yield stress fluid is displaced by a low-viscosity Newtonian one.

Recently, Rodríguez de Castro and Radilla [2016a] conducted non-Darcian flow experiments of shear-thinning fluids without yield stress in rough-walled fractures, showing that the inertial pressure losses do not depend on fluid's rheology. These authors proposed a method to predict the pressure losses generated during non-Darcian shear-thinning flow from the values of $\mathrm{K}, \gamma$ and $\beta$ obtained during creeping Newtonian flow and the shear-viscosity parameters of the fluid. Their predictions showed good agreement with experimental data. However, a major drawback was the use of an experimentally obtained shift parameter to relate the apparent viscosity of the fluid in the porous medium to its bulk viscosity. Indeed, the calculation of the mentioned shift parameter involved carrying out preliminary Darcian shear-thinning flow experiments, so predicting its value is of considerable interest. Another interesting prospect consisted in extending this prediction method to the case of shearthinning fluids with yield stress. The same authors also proposed a simple method to predict non-Darcian flow of Carreau fluids through packed beads [Rodríguez de Castro and Radilla, 
2016b]. However, the flow of yield stress fluids was not tackled in these works and no estimate of the shift parameter relating the apparent viscosity of the fluid in the porous medium to its bulk viscosity was provided either.

Many applications require the flow rate in a fracture to be predicted from the applied pressure gradient and known fracture size and fluid rheology. In particular, understanding the flow of drilling fluids with yield stress through a rough-walled fracture is of vital importance in order to design the additives used to stop the fluid loss when a fracture is hit during drilling [Lavrov, 2013]. In this work, a simple approach is proposed to extend Darcy's and full cubic laws to the case of yield stress and Carreau fluids. In order to achieve this goal, flow experiments with concentrated aqueous polymer solutions have been conducted using replicas of natural fractures. Particular attention will be paid to investigating how yield stress affects the relationships between flow rate and pressure losses in rough-walled rock fractures.

\section{Predicting the flow of yield stress fluids and Carreau fluids in porous media}

The shear-thinning behaviour of semi-dilute polymer solutions widely used in EOR and soil remediation is commonly represented by the empirical Carreau model [Carreau, 1972] based on molecular network theory [Sorbie, 1989; López et al., 2003; Rodríguez de Castro et al.,

2016b]. The Carreau equation is often presented as $\frac{\mu-\mu_{\infty}}{\mu_{0}-\mu_{\infty}}=\left[1+(\lambda \dot{\gamma})^{2}\right]^{\frac{n-1}{2}}$, where $\mu$ is the viscosity at a given shear rate $\dot{\gamma}, \mu_{0}$ and $\mu_{\infty}$ are the zero shear rate and infinite shear rate viscosities, respectively, $\mathrm{n}$ is the power-law index, and $\lambda$ is the time constant. $\mathrm{n}$ is inferior to unity for shear-thinning fluids. The values of $\mu_{0}, \mu_{\infty}, \mathrm{n}$ and $\lambda$ are determined by the polymer 
concentration under given pressure and temperature conditions. In the region far from the low

262 shear viscosity plateau, i.e. when $\dot{\gamma} \gg \frac{1}{\lambda}$, Carreau's law leads to the following expression

$\mu \approx \mu_{\infty}+\left(\mu_{0}-\mu_{\infty}\right) \lambda^{\mathrm{n}-1} \dot{\gamma}^{\mathrm{n}-1}=\mu_{\infty}+\mathrm{c} \dot{\gamma}^{\mathrm{n}-1}$

264 with $c=\left(\mu_{0}-\mu_{\infty}\right) \lambda^{\mathrm{n}-1}$. Given that all the shear rates involved in the flow experiments with 265 Carreau fluids analysed in this work are sufficiently high, only the high shear rates version of 266 Carreau's equation (Eq. 9) will be considered subsequently.

Some concentrated polymer solutions present a yield stress, as shown in previous works [Song et al., 2006; Carnali, 1991; Withcomb and Macosko, 1978; Economides and Nolte, 2000; Khodja, 2008; Benmouffok-Benbelkacem et al., 2010]. The steady-state shear flow of concentrated polymer solutions has been proved to be well described by the HerschelBulkley law [Herschel and Bulkley, 1926]. This empirical law can be written as follows:

272

$\left\{\begin{array}{ccc}\tau=\tau_{0}+a \dot{\gamma}^{n} & \text { for } & \tau>\tau_{0} \\ \dot{\gamma}=0 & \text { for } & \tau \leq \tau_{0}\end{array}\right.$

273 where $\tau_{0}$ is the yield stress, $a$ is the consistency and $n$ is the flow index of the fluid. In the case of shear-thinning yield stress fluids, $\mathrm{n}$ is inferior to unity. The three parameters are generally obtained by fitting the data obtained by measuring the shear rate $\dot{\gamma}$ as a function of the applied shear stress $\tau$ using a rheometer.

278 A practical approach to study the flow of complex fluids with shear-rate-dependent viscosity through a porous medium consists in defining an equivalent viscosity $\mu_{\mathrm{eq}}$ as being the 
quantity that must replace the viscosity in Darcy's law to result in the same pressure drop actually measured [Tosco et al., 2013]. In the case of a rectangular fracture, $\mu_{\mathrm{eq}}$ is expressed as:

$\mu_{\mathrm{eq}}=\mathrm{K} \frac{\nabla \mathrm{P}}{\mathrm{u}}=\frac{\mathrm{h}^{2}}{12} \frac{\nabla \mathrm{P}}{\mathrm{u}}$

284 It should be noted that both inertial and viscous effects are encompassed in $\mu_{\text {eq. }}$. In order to analyse the viscous effects separately, the "in situ" shear viscosity $\mu_{\mathrm{pm}}$ in the porous medium must be calculated. To do such calculation from the constitutive equation of the fluid, an apparent shear rate in the porous medium has to be determined first. The apparent shear rate $\dot{\gamma}_{\mathrm{pm}}$ of shear-thinning fluids flowing through a porous medium can be defined by dividing the mean velocity $\mathrm{u}$ by a characteristic microscopic length of the porous media [Chauveteau, 1982; Sorbie et al., 1989; Perrin et al., 2006; Tosco et al., 2013; Rodríguez de Castro et al., 2016b]. This microscopic length is usually taken as $\sqrt{\mathrm{K} \varepsilon}$ with $\varepsilon$ being the porosity of the porous medium. From the definition of cross-sectional area (Eq. 7), it is expected that porosity is close to unity in the particular case of fractures. Therefore, $\dot{\gamma}_{\mathrm{pm}}$ can be defined as:

$\dot{\gamma}_{\mathrm{pm}}=\alpha \frac{\mathrm{u}}{\sqrt{\mathrm{K}}}=\alpha \frac{2 \sqrt{3} \mathrm{u}}{\mathrm{h}}$

where $\alpha$ is a empirical shift factor known to be a function of both the bulk rheology of the fluid and the porous media [Chauveteau, 1982; Sorbie et al., 1989; López et al., 2003; López, 2004; Comba et al., 2011]. Previous research showed that $\dot{\gamma}_{\mathrm{pm}}$ corresponds to the wall shear 
$\mu_{\mathrm{pm}, \text { Carreau }}=\mu_{\infty}+\mathrm{c}\left(\alpha \frac{2 \sqrt{3} \mathrm{u}}{\mathrm{h}}\right)^{\mathrm{n}-1}$ Sheng, 2011]. from Eqs. (9) and (12):

rate in the average pore throat diameter [Chauveteau and Zaitoun, 1981; Chauveteau, 1982;

The usual approach to determine the value $\alpha$ consists in overlaying the porous medium $\mu_{\mathrm{eq}}$ vs. $\dot{\gamma}_{\text {app }}$ with the bulk $\mu_{\text {eq }}$ vs. $\dot{\gamma}$ curves as closely as possible and noting the scale change in shear rate required to obtain the best fit. This criterion to select $\alpha$ was proposed by Sorbie et al. [1989] as a pragmatic alternative to the original one previously proposed by Chauveteau [1982], and was subsequently used by other authors [González et al., 2005; Amundarain et al., 2009]. It should be noted that a good overlay between both curves is only possible in the low flow rates region where no significant inertial effects occur, assuming no wall slip [Tosco et al., 2013; Rodríguez de Castro and Radilla, 2016a]. Keeping in mind the objective to propose a prediction method, expressions for the calculation of $\alpha$ must be provided so as to avoid the need to perform $\alpha$-determination experiments.

In the case of Carreau fluids flowing at moderate and high shear rates $\mu_{\mathrm{pm}}$ can be obtained 
$\mu_{\mathrm{pm}, \mathrm{ysf}}=\frac{\tau_{0} \mathrm{~h}}{\alpha 2 \sqrt{3} \mathrm{u}}+\mathrm{a}\left(\alpha \frac{2 \sqrt{3} \mathrm{u}}{\mathrm{h}}\right)^{\mathrm{n}-1}$

320 Although Eqs. (12-14), which are based on the bundle-of-capillaries model, were originally proposed for the flow of non-Newtonian fluids through packed beads, the apparent viscosity was found to correlate reasonably well in porous media with complex pre geometry and topology [Sorbie et al., 1989].

Let us focus now on the determination of the wall shear rate in rectangular channels. For the steady 2D-flow of an incompressible fluid through a rectangular channel, the wall shear stress $\tau_{\mathrm{w}}$ is related to the pressure gradient $\nabla \mathrm{P}$ as follows [Pipe et al., 2008]:

$$
\tau_{\mathrm{w}}=\frac{\mathrm{wh}}{2(\mathrm{w}+\mathrm{h})} \nabla \mathrm{P}
$$

329 For the calculation of $\nabla \mathrm{P}$ in Eq. (15), the fractures will be modelled as being rectangular channels of width w and depth $\mathrm{h}$. As explained above, $\dot{\gamma}_{\mathrm{pm}}$ corresponds to the wall shear rate in the average pore throat diameter [Chauveteau and Zaitoun, 1981; Chauveteau, 1982; Sheng, 2011]. In the case of a rough-walled fracture, the average pore throat diameter can be assimilated to the hydraulic aperture. Therefore, $\dot{\gamma}_{\mathrm{pm}}$ can be interpreted as the wall shear rate in a section of aperture $\mathrm{h}$. The wall shear stress in a section of aperture $\mathrm{h}$ can be calculated from Eq. (15), by using Eqs. (1) and (13) for the case of Carreau fluids: 


$$
\tau_{w}=\frac{6 w}{(w+h)} \frac{u}{h}\left[\mu_{\infty}+c \dot{\gamma}_{p m}^{n-1}\right]=\frac{6 w}{(w+h)} \frac{u}{h}\left[\mu_{\infty}+2^{n-1} 3^{\frac{n-1}{2}} c\left(\alpha \frac{u}{h}\right)^{n-1}\right]
$$

339 And using Eqs. (1) and (14) for the case of Herschel-Bulkley fluids, Eq. (15) can be written 340 as:

$$
\tau_{w}=\frac{6 w}{(w+h)} \frac{u}{h}\left(\frac{\tau_{0}}{\dot{\gamma}_{p m}}+a \dot{\gamma}_{p m}^{n-1}\right)=\frac{\sqrt{3} w}{(w+h) \alpha}\left[\tau_{0}+2^{n} 3^{\frac{n}{2}} a\left(\alpha \frac{u}{h}\right)^{n}\right]
$$

342 For a constant viscosity fluid, the wall shear rate is given by $\dot{\gamma}_{\mathrm{w}, \text { Newtonian }}=\frac{6 \mathrm{u}}{\mathrm{h}}$. However, for incompressible flows of liquids with a shear-rate-dependent viscosity, the calculation of $\dot{\gamma}_{\mathrm{w}}$ is more complex given that the velocity profile is no longer parabolic [Pipe et al., 2008]. An apparent shear rate $\dot{\gamma}_{\text {app }}$ can thus be defined as:

$$
\dot{\gamma}_{\mathrm{app}}=\frac{6 \mathrm{u}}{\mathrm{h}}
$$
equation [Macosko, 1994; Pipe et al., 2008]: 
$\dot{\gamma}_{\mathrm{w}}=\frac{\dot{\gamma}_{\mathrm{app}}}{3}\left[2+\frac{\mathrm{d}\left(\ln \dot{\gamma}_{\mathrm{app}}\right)}{\mathrm{d}\left(\ln \tau_{\mathrm{w}}\right)}\right]$

351

352 Therefore, the next equation can be obtained from Eqs. (16), (18) and (19) for a Carreau

353 fluid:

$\dot{\gamma}_{\mathrm{w}, \text { Carreau }}=\frac{2 \mathrm{u}}{\mathrm{h}}\left(2+\frac{2 \sqrt{3} \alpha \mathrm{h}^{\mathrm{n}} \mu_{\infty} \mathrm{u}+2^{\mathrm{n}} 3^{\mathrm{n} / 2} \mathrm{ah}(\alpha u)^{\mathrm{n}}}{2 \sqrt{3} \alpha \mathrm{h}^{\mathrm{n}} \mu_{\infty} \mathrm{u}+2^{\mathrm{n}} 3^{\mathrm{n} / 2} \mathrm{ahn}(\alpha u)^{\mathrm{n}}}\right)$

355 Analogously, the next equation can be obtained from Eqs. (17), (18) and (19) for a Herschel-

356 Bulkley fluid:

$\dot{\gamma}_{\mathrm{w}, \mathrm{ysf}}=\frac{2 \mathrm{u}}{\mathrm{h}}\left(2+\frac{\mathrm{a}+2^{-\mathrm{n}} 3^{-\mathrm{n} / 2} \tau_{0}\left(\alpha \frac{\mathrm{u}}{\mathrm{h}}\right)^{-\mathrm{n}}}{\mathrm{an}}\right)$

357

358

For a Carreau fluid, $\dot{\gamma}_{\mathrm{pm}}=\dot{\gamma}_{\mathrm{w}, \text { Carreau }}$, so Eqs. (12) and (20) lead to the following expression:

359

$\alpha=\frac{6 \sqrt{3} \alpha h^{n} \mu_{\infty} u+2^{n} 3^{\frac{n}{2}} a h(1+2 n)(\alpha u)^{n}}{6 \alpha h^{n} \mu_{\infty} u+2^{n} 3^{\frac{n+1}{2}} a h n(\alpha u)^{n}}$

360

361 In the case of a Herschel-Bulkley fluid ( $\left.\dot{\gamma}_{\mathrm{pm}}=\dot{\gamma}_{\mathrm{w}, \mathrm{ysf}}\right)$, Eqs. 12 and 21 lead to: 


$$
\alpha=\frac{1}{\sqrt{3}}\left(2+\frac{\mathrm{a}+2^{-\mathrm{n}} 3^{-\mathrm{n} / 2} \tau_{0}\left(\alpha \frac{\mathrm{u}}{\mathrm{h}}\right)^{-\mathrm{n}}}{\mathrm{an}}\right)
$$

364 From Eqs. (22) and (23), it can be deduced that $\alpha$ is not a constant parameter in the case of 365 Carreau fluids and yield stress fluids, but depends on u. For the simpler case of a power-law 366 fluid ( $\left.\tau_{0}=0\right)$, Eq. (23) leads to:

367

$$
\alpha=\frac{1}{\sqrt{3}}\left(2+\frac{1}{n}\right)
$$

368 which becomes $\alpha=\sqrt{3}$ for a Newtonian fluid. Therefore, $\alpha$ is a constant parameter only if $\tau_{0}=0$ and $\mu_{\infty}=0$

371 Given that $\alpha$ depends on $\mathrm{u}$ for both Carreau and yield stress fluids, Eqs. (22) and (23) are

372 only relevant in the cases $\mu_{\infty}=0$ and $\tau_{0}=0$, respectively. For $\mu_{\infty} \neq 0$ and $\tau_{0} \neq 0$, Eq. (19) becomes:

$$
\dot{\gamma}_{\mathrm{w}}=\frac{\dot{\gamma}_{\mathrm{app}}}{3}\left[2+\frac{\frac{\mathrm{d}\left(\ln \dot{\gamma}_{\mathrm{app}}\right)}{\mathrm{du}} \mathrm{du}}{\frac{\partial\left(\ln \tau_{\mathrm{w}}\right)}{\partial \mathrm{u}} \mathrm{du}+\frac{\partial\left(\ln \tau_{\mathrm{w}}\right)}{\partial \alpha} \mathrm{d} \alpha}\right]=\frac{\dot{\gamma}_{\mathrm{app}}}{3}\left[2+\frac{\frac{\mathrm{d}\left(\ln \dot{\gamma}_{\mathrm{app}}\right)}{\mathrm{du}}}{\frac{\partial\left(\ln \tau_{\mathrm{w}}\right)}{\partial \mathrm{u}}+\frac{\partial\left(\ln \tau_{\mathrm{w}}\right)}{\partial \alpha} \frac{\mathrm{d} \alpha}{\mathrm{du}}}\right]
$$

where $\alpha$ is a function of $u$. 
Therefore, for a Carreau fluid, Eq. (20) becomes:

$$
\dot{\gamma}_{\mathrm{w}, \text { Carreau }}=\frac{2 \mathrm{u}}{\mathrm{h}}\left[2+\frac{\alpha\left(2 \sqrt{3} \mu_{\infty} \mathrm{u} \alpha+2^{\mathrm{n}} 3^{\frac{\mathrm{n}}{2}} \mathrm{ah}\left(\frac{\mathrm{u} \alpha}{\mathrm{h}}\right)^{\mathrm{n}}\right)}{2 \sqrt{3} \mu_{\infty} \alpha^{2}+2^{\mathrm{n}} 3^{\frac{\mathrm{n}}{2}} \mathrm{ahn} \alpha\left(\frac{\mathrm{u} \alpha}{\mathrm{h}}\right)^{\mathrm{n}}+2^{\mathrm{n}+1} 3^{\frac{\mathrm{n}}{2}} \mathrm{ah}(\mathrm{n}-1) \mathrm{u}\left(\frac{\mathrm{u} \alpha}{\mathrm{h}}\right)^{\mathrm{n}} \frac{\partial \alpha}{\partial \mathrm{u}}}\right]
$$

379 Also, for a Herschel-Bulkley fluid, Eq. (21) becomes:

380

$$
\dot{\gamma}_{\mathrm{w}, \mathrm{ysf}}=\frac{2 \mathrm{u}}{\mathrm{h}}\left[2+\frac{\alpha\left(\tau_{0}+2^{\mathrm{n}} 3^{\frac{\mathrm{n}}{2} \mathrm{a}}\left(\frac{\mathrm{u} \alpha}{\mathrm{h}}\right)^{\mathrm{n}}\right)}{2^{\mathrm{n}} 3^{\frac{\mathrm{n}}{2}} \mathrm{an} \alpha\left(\frac{\mathrm{u} \alpha}{\mathrm{h}}\right)^{\mathrm{n}}-2 \mathrm{u}\left(\tau_{0}-2^{\mathrm{n}} 3^{\frac{\mathrm{n}}{2}} \mathrm{a}(\mathrm{n}-1)\left(\frac{\mathrm{u} \alpha}{\mathrm{h}}\right)^{\mathrm{n}}\right) \frac{\partial \alpha}{\partial \mathrm{u}}}\right]
$$

Consequently, the following differential equation has to be solved in order to determine $\alpha$ as

$$
\alpha=\frac{1}{\sqrt{3}}\left[2+\frac{\alpha\left(2 \sqrt{3} \mu_{\infty} u \alpha+2^{\mathrm{n}} 3^{\frac{\mathrm{n}}{2}} \mathrm{ah}\left(\frac{\mathrm{u} \alpha}{\mathrm{h}}\right)^{\mathrm{n}}\right)}{2 \sqrt{3} \mu_{\infty} \mathrm{u} \alpha^{2}+2^{\mathrm{n}} 3^{\frac{\mathrm{n}}{2}} \mathrm{ahn} \alpha\left(\frac{\mathrm{u} \alpha}{\mathrm{h}}\right)^{\mathrm{n}}+2^{\mathrm{n}+1} 3^{\frac{\mathrm{n}}{2}} \mathrm{ah}(\mathrm{n}-1) \mathrm{u}\left(\frac{\mathrm{u} \alpha}{\mathrm{h}}\right)^{\mathrm{n}} \frac{\partial \alpha}{\partial \mathrm{u}}}\right]
$$

And for a Herschel-Bulkley fluid:

387

$$
\alpha=\frac{1}{\sqrt{3}}\left[2+\frac{\alpha\left(\tau_{0}+2^{\mathrm{n}} 3^{\frac{\mathrm{n}}{2}} \mathrm{a}\left(\frac{\mathrm{u} \alpha}{\mathrm{h}}\right)^{\mathrm{n}}\right)}{2^{\mathrm{n}} 3^{\frac{\mathrm{n}}{2}} \mathrm{an} \alpha\left(\frac{\mathrm{u} \alpha}{\mathrm{h}}\right)^{\mathrm{n}}-2 \mathrm{u}\left(\tau_{0}-2^{\mathrm{n}} 3^{\frac{\mathrm{n}}{2}} \mathrm{a}(\mathrm{n}-1)\left(\frac{\mathrm{u} \alpha}{\mathrm{h}}\right)^{\mathrm{n}}\right) \frac{\partial \alpha}{\partial \mathrm{u}}}\right]
$$

Eqs. (29) and (30) can be numerically solved within a given range of $u$ to obtain the relation between $\alpha$ and $u$. Then, the obtained relation can be used in Eq. (13) for a Carreau fluid and in Eq. (14) for a Herschel-Bulkley fluid to obtain $\mu_{\mathrm{pm} \text {,Carreau }}$ and $\mu_{\mathrm{pm} \text {,ysf }}$, respectively. Once 


$$
\nabla \mathrm{P}=\frac{\mu_{\mathrm{pm}}}{\mathrm{K}} \mathrm{u}=\frac{12 \mu_{\mathrm{pm}}}{\mathrm{h}^{2}} \mathrm{u}
$$

396

$$
\nabla \mathrm{P}=\frac{\mu_{\mathrm{pm}}}{\mathrm{K}} \mathrm{u}+\beta \rho \mathrm{u}^{2}=\frac{12 \mu_{\mathrm{pm}}}{\mathrm{h}^{2}} \mathrm{u}+\beta \rho \mathrm{u}^{2}
$$

$$
\nabla P=\frac{\mu_{\mathrm{pm}}}{\mathrm{K}} \mathrm{u}+\beta \rho \mathrm{u}^{2}+\frac{\gamma \rho^{2}}{\mu_{\mathrm{pm}}} \mathrm{u}^{3}=\frac{12 \mu_{\mathrm{pm}}}{\mathrm{h}^{2}} \mathrm{u}+\beta \rho \mathrm{u}^{2}+\frac{\gamma \rho^{2}}{\mu_{\mathrm{pm}}} \mathrm{u}^{3}
$$

399 It is remarked that $\frac{12 \mu_{\mathrm{pm}}}{\mathrm{h}^{2}} \mathrm{u}=\frac{2 \sqrt{3} \tau_{0}}{\mathrm{~h}} \frac{1}{\alpha}+\frac{2^{\mathrm{n}+1} 3^{\frac{\mathrm{n}+1}{2}} \mathrm{a}}{\mathrm{h}^{\mathrm{n}+1}} \alpha^{\mathrm{n}-1} \mathrm{u}^{\mathrm{n}}=\mathrm{C}_{1} \frac{1}{\alpha}+\mathrm{C}_{2} \alpha^{\mathrm{n}-1} \mathrm{u}^{\mathrm{n}}$ in the case of 400 Herschel-Bulkley fluids, with $C_{1}=\frac{2 \sqrt{3} \tau_{0}}{h}$ and $C_{2}=\frac{2^{n+1} 3^{\frac{n+1}{2}} a}{h^{n+1}}$. Therefore, Eqs. (31) to (33) 401 present a limiting pressure gradient [Roustaei et al., 2016] of value $\mathrm{C}_{1} \frac{1}{\alpha}$. In the preceding 402 expressions, $\mu_{\mathrm{pm}}$ corresponds to $\mu_{\mathrm{pm} \text {,Carreau }}$ or $\mu_{\mathrm{pm}, \mathrm{ysf}}$ depending on the type of fluid being 403 considered.

404 
407 It should be noted that elongational flows during the injection of solutions of polymers 408 presenting a certain degree of flexibility through porous media are known to induce extra 409 pressure losses with respect to pure shear flow [Rodríguez et al,. 1993; Müller and Sáez, 1999; Nguyen and Kausch, 1999; Seright et al., 2011; Amundarain et al., 2009). These extra pressure losses were attributed to the formation of transient entanglements of polymer molecules due to the action of the extensional component of the flow. In the present approach, we first hypothesize that the differences between the total pressure drops measured during the flow of the investigated complex fluids through rough-walled rock fractures and the viscous pressure drop as predicted from the shear viscosity of the fluid can be explained in terms of inertial effects generated in the porous medium flow. This hypothesis is then validated through analysis of the experimental results.

\section{Materials and Methods}

In this section, we present the experimental procedure and the materials used to carry out the flow experiments with a yield stress fluid specifically performed in the framework of the present study. However, the proposed method to predict $\nabla \mathrm{P}$ as a function of $\mathrm{u}$ in roughwalled fractures is also compared with previously presented experimental data [Rodríguez de Castro and Radilla, 2016a] in order to assess its efficiency in the case of Carreau fluids. 
429 A series of experiments was conducted injecting a concentrated aqueous polymer solution 430 through two transparent epoxy resin replicas of natural rough-walled rock fractures. The

431

432 original fractures used in this work are a Vosges sandstone sample with dimensions $26 \mathrm{~cm}$ long and $\mathrm{w}=14.8 \mathrm{~cm}$ wide, and a granite sample with dimensions $33 \mathrm{~cm}$ long and $\mathrm{w}=15.5$ $\mathrm{cm}$ wide. Details of the fabrication process of these fracture replicas can be found elsewhere [Isakov et al., 2001; Nowamooz et al., 2013]. The aperture maps of both fractures obtained by Nowamooz et al. [2013] have been included as supporting information of the present article (Figures $S_{1}$ and $S_{2}$ ), showing the high spatial variability. The latter authors analysed in detail the aperture variability and distribution of the fractures using an image processing procedure based on the attenuation law of Beer-Lamber. They showed that the smallest apertures are located at the centre and the largest apertures are located near the inlet and the outlet of the fractures. Moreover, the apertures of the Vosges sandstone fracture are more variable at lower half than at the upper half, while the spatial variability appears to be relatively high across the entire granite fracture area. This results in a more heterogeneous aperture map for the granite fracture. Moreover, Nowamooz et al. [2013] showed that the spatial variability of the fracture aperture field, especially the constricted areas at the centre of the fractures, resulted in the creation preferential paths for the flow of the fluid. These effects are expected to be more important in the case of shear-thinning fluids and yield stress fluids as the pressure loss sensitivity to aperture is higher (shear viscosity depends on the local aperture) [Roustaei et al., 2016].

Two different configurations were used depending on the involved flow rates. For the lowest flow rates, ranging from $0.06 \mathrm{~L} / \mathrm{h}$ to $6 \mathrm{~L} / \mathrm{h}$, the injection circuit was open and the fluid was injected through the fractures at the selected flow rate using a dual piston pump (Prep Digital HPCL pump, A.I.T., France). For the highest flow rates, ranging from $9 \mathrm{~L} / \mathrm{h}$ to $250 \mathrm{~L} / \mathrm{h}$, the 
circuit was closed. In this case, the fluid was injected from a tank situated upstream of the

455

456

457

458

459

460

461

462

463

464

465

466

467 fracture using a volumetric pump (EcoMoineau M Series, PCM, France), and its flow rate was measured with a positive displacement flow meter (Model LSM45, Oval, Japan). The injected fluid was continuously recirculated to the upstream tank after passing through the fracture. A differential pressure sensor (DP15 Variable Reluctance Pressure Sensor, Validyne, USA) was used to measure the pressure drop over a distance of $\mathrm{L}=20.5 \mathrm{~cm}$ in the case of the Vosges sandstone fracture and $\mathrm{L}=27 \mathrm{~cm}$ in the case of the granite fracture. A sketch of the experimental setup is shown in Figure 1. The range of the piston pump was from $6 \times 10^{-3}$ to $6 \mathrm{~L} / \mathrm{h}$ with an accuracy of $\pm 2 \%$ while the volumetric pump was able to provide flow rates ranging from 0 to $300 \mathrm{~L} / \mathrm{h}$. The range of the flow meter installed at the outlet of the volumetric pump was from 7 to $500 \mathrm{~L} / \mathrm{h}$ with an accuracy of $\pm 1 \%$ and the range of the pressure sensor was adjusted by installing different membranes from 0-1400 Pa to 0$56000 \mathrm{~Pa}$ with an accuracy of $\pm 0.3 \%$ of the full scale

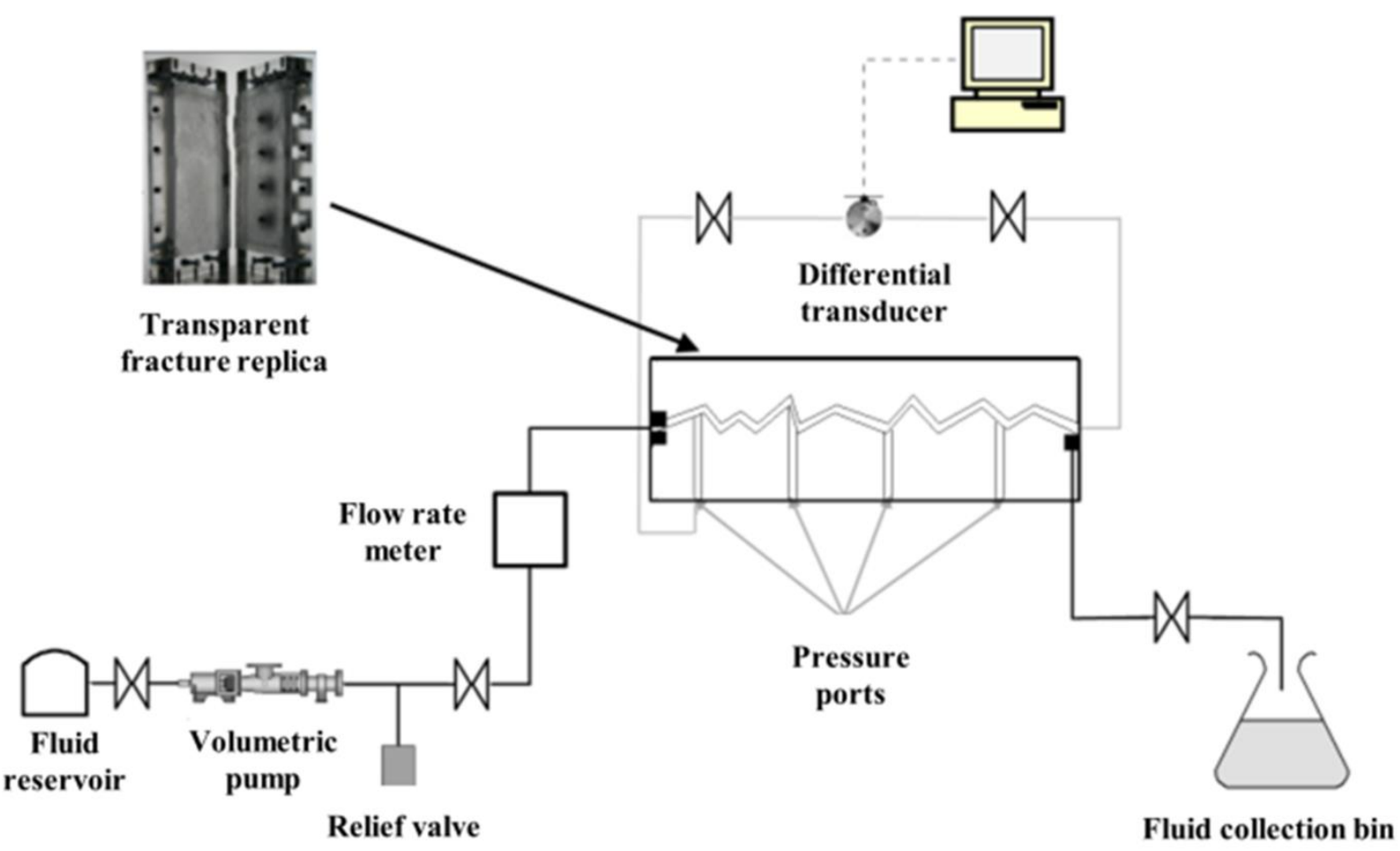

Figure 1. Sketch of the experimental setup used in the present experiments. 
471 The procedure followed in our experiments was similar to the one followed by Rodríguez de

472 Castro and Radilla [2016a], but the covered range of injection flow rates was considerably

473 wider. In this procedure, the fractures were saturated with $\mathrm{CO}_{2}$ (more water-miscible gas than

474 air) prior to saturation with polymer solution in order to avoid air trapping during the experiments. Once saturated with polymer solution, a set of forty-five different flow rates ranging from 0.06 to $250 \mathrm{~L} / \mathrm{h}$ were imposed for the flow through the fracture and the corresponding pressure drops were measured. It can be observed that the range of $u$ used in this work is significantly wider than those used in some preceding works (Sabiri and Comiti, 1994), which permits a better assessment of the proposed prediction methods (over $~ 3.6$ orders of magnitude). Each step was repeated four times and the uncertainty related to the repeatability of the pressure drop and the accuracy of the involved instruments was calculated as $\pm 2 \sigma$, with $\sigma$ being an estimate of the relative standard deviation of the measurements $(95 \%$ confidence interval). The room temperature during the experiments was $20^{\circ} \mathrm{C} \pm 1$.

\subsection{Fluid Properties}

Filtered water and a xanthan gum aqueous solution with polymer concentration $C_{p}=7000$ ppm were used as injected fluid in the present experiments. Xanthan gum is an important industrial biopolymer commonly obtained through fermentation of Xanthomonas campestris bacteria [Garcia-Ochoa et al., 2000; Palarinaj and Javarman, 2011; Wadhai and Dixit, 2011]. This biopolymer is widely used as viscosity-enhancing additive in the food and cosmetics industries, as zerovalent iron for groundwater remediation and as part of the formulation of drilling muds in EOR [Garcia-Ochoa et al., 2000; Amundarain et al., 2009; 
Palarinaj and Javarman, 2011; Wadhai and Dixit, 2011; Xin et al., 2015]. In solution state,

495

496

497

498

499

500

501

502

503

504

505

506

507

508

509

510

511

512

513

514

515

516

517

518

an isolated xanthan macromolecule is more or less rigid and is of typically $1 \mu \mathrm{m}$ of contour length [Mongruel and Cloitre, 2003] and a transverse size of approximately $2 \mathrm{~nm}$. Song [2007] presented additional information about the chemical composition, structure and other physico-chemical properties of this biopolymer. Xanthan gum solutions are one of the main examples of inelastic, shear-thinning fluids in contrast to linear flexible polymers as polyacrylamide [Jones andWalters 1989; Sorbie 1991a] which are highly viscoelastic. Due to the stiffness of its molecule, xanthan semidilute aqueous solutions develop a high viscosity level and a very pronounced shear-thinning behavior. Therefore, xanthan gum solutions have been reported to present an apparent yield stress [Song et al., 2006; Carnali, 1991;Withcomb and Macosko, 1978; Khodja, 2008; Benmouffok-Benbelkacem et al., 2010] even if strictly speaking, they should be referred to as pseudo-yield stress fluids. The Herschel-Bulkley model [Herschel and Bulkley, 1926] has been proved to describe the steady-state shear flow of concentrated xanthan gum solutions [Song et al., 2006; Rodríguez de Castro et al., 2014, 2016a].

Sixty litres of polymer solution were prepared by dissolving xanthan gum in filtered water containing $400 \mathrm{ppm}$ of $\mathrm{NaN}_{3}$ as a bactericide. The xanthan gum powders were progressively dissolved in water while gently mixing with a custom-made overhead device. Once prepared, the polymer solution was characterized by means of a stress controlled rheometer (ARG2, TA Instruments) equipped with cone-plate geometry at a constant temperature of $19^{\circ} \mathrm{C} \pm 1$, following a procedure previously presented in the literature [Rodríguez de Castro et al., 2014, 2016a, 2016b]. The obtained rheograms are provided as supporting information (Figure S3). Eq. (10) was used to fit the rheograms following the procedure presented by Rodríguez de Castro et al. [2014] and obtaining $\tau_{0}=7.4 \mathrm{~Pa}, \mathrm{a}=0.37 \mathrm{~Pa} \mathrm{~s}^{\mathrm{n}}$ and $\mathrm{n}=0.52$. A viscosity of 
$0.001 \mathrm{~Pa}$ s was measured for the solvent (water) and the densities $\rho$ of both the water and the xanthan gum solution were taken as $1000 \mathrm{~kg} / \mathrm{m}^{3}$.

Moreover, a set of effluent fluid samples were collected at the outlet of the fractures after injection at the highest flow rate. The effluent rheograms were determined and compared to that of the inflowing fluid in order to assess polymer degradation and retention of the polymer on the fracture walls. No significant difference was observed between the rheograms, so polymer degradation and significant polymer retention were proved to be negligible. Moreover, no air macro bubbles were observed in the injected fluid. Also, the rheograms of a degassed fluid sample and an undegassed fluid sample were measured and compared in order to evaluate the influence of residual air micro bubbles, showing no significant difference.

\section{The Carreau fluids used in the non-Darcian shear-thinning flow experiments in rough-walled} fractures performed by [Rodríguez de Castro and Radilla, 2016a] were three xanthan gum aqueous solutions with polymer concentrations of $200 \mathrm{ppm}, 500 \mathrm{ppm}$ and $700 \mathrm{ppm}$, respectively. The corresponding rheological parameters used in Eq. (9) for these fluids were $\left[\mathrm{c}=4.8 \times 10^{-3} \mathrm{~Pa} \mathrm{~s}^{\mathrm{n}}, \mu_{\infty}=1.1 \times 10^{-3} \mathrm{~Pa} \mathrm{~s}, \mathrm{n}=6.6 \times 10^{-1}\right]$ for $\mathrm{C}_{\mathrm{p}}=200 \mathrm{ppm},\left[\mathrm{c}=2.4 \times 10^{-3} \mathrm{~Pa}\right.$ $\left.\mathrm{s}^{\mathrm{n}}, \mu_{\infty}=1.1 \times 10^{-3} \mathrm{~Pa} \mathrm{~s}, \mathrm{n}=5.8 \times 10^{-1}\right]$ for $\mathrm{C}_{\mathrm{p}}=500 \mathrm{ppm}$ and $\left[\mathrm{c}=4.2 \times 10^{-3} \mathrm{~Pa} \mathrm{~s}^{\mathrm{n}}, \mu_{\infty}=1.1 \times\right.$ $10^{-3}$ Pa s, $\left.\mathrm{n}=5.2 \times 10^{-1}\right]$ for $\mathrm{C}_{\mathrm{p}}=700 \mathrm{ppm}$. 
542 The flow experiments were conducted for both fluids (water and yield stress fluid) and were repeated four times. For each fluid, a total of a hundred and eighty (four repetitions for each of the forty-five flow rates) were completed. The hundred and eighty measures for a given fluid-fracture pair were considered to be an experimental set.

\subsection{Non-Darcian flow of a Newtonian fluid: obtaining $K, \gamma$ and $\beta$ from experiments}

The experimental sets of $\nabla \mathrm{P}$ as a function of $\mathrm{u}$ for water injection $\left(\mathrm{C}_{\mathrm{p}}=0 \mathrm{ppm}\right)$ through both fractures are included as supporting information (Figure S4). Higher pressure losses were obtained for the less permeable fracture (Vosges sandstone), as expected, and non-linear relations between $\mathrm{u}$ and $\mathrm{\nabla P}$ were observed in both cases steaming from inertial effects at high flow rates. It is known that directly fitting Eq. (5) to the whole set of data results in overestimation of permeability [Du Plessis and Masliyah, 1988; Dukhan et al., 2014]. Indeed, by fitting the whole set of data to the polynomial law, a part of the pressure drop would be attributed to inertial effects even at the lowest flow rates, which is not realistic. Consequently, the viscous pressure loss would be underestimated leading to permeability overestimation. To avoid this issue, the procedure proposed by Rodríguez de Castro and Radilla [2016a] was followed to determine $\mathrm{h}$ and $\mathrm{K}$ in the present experiments. This procedure is divided into two-steps:

1) In this step, the hydraulic apertures $h_{j}$ obtained by only using the first $j$ experimental data

562 (starting with the lowest flow rates) are calculated by minimizing the sum $\sum_{\mathrm{i}=1}^{\mathrm{j}}\left(\nabla \mathrm{P}_{\mathrm{i}}-\right.$ 
$\left.563 \frac{12 Q_{i} \mu}{h_{j}^{3} w}\right)^{2}$ for $\mathrm{j}=1 \ldots \mathrm{N}$, with $\mathrm{N}$ being the number of experimental data and $\mu$ being the 564 measured dynamic viscosity of water at the room temperature (0.001 Pa s).

566 2) Then, the quality of the $\mathrm{N}$ fits obtained by using the $\mathrm{N}$ values of $h_{j}$ calculated in the

567 preceding step is evaluated by using the merit function $F(j)=\frac{\sum_{i=1}^{j}\left|\frac{\nabla \mathrm{P}_{i}-\frac{12 Q_{j} \mu}{h_{j}^{3} w}}{\nabla P_{i}}\right|}{j}$ for $j=1 \ldots N$.

568 After that, the value of $\mathrm{j}$ minimizing $\mathrm{F}(\mathrm{j})$ was determined. The corresponding $\mathrm{h}_{\mathrm{j}}$ value was 569 selected as the hydraulic aperture of the fracture from which K was calculated using Eq. (7).

570

571 The obtained values for the granite fracture were $\mathrm{K}=6.1 \times 10^{-8} \mathrm{~m}^{2}( \pm 2 \%)$ and $\mathrm{h}=8.5 \times 10^{-4}$ $572 \mathrm{~m}( \pm 2 \%)$, while for the Vosges sandstone fracture the computed values were $\mathrm{K}=2.1 \times 10^{-8}$ $573 \mathrm{~m}^{2}( \pm 1 \%)$ and $\mathrm{h}=5.0 \times 10^{-4} \mathrm{~m}( \pm 1 \%)$. Once permeability was determined, the $\left(\mathrm{Q}_{\mathrm{i}}, \nabla \mathrm{P}_{\mathrm{i}},\right)$ data were fitted to a full cubic law (Eq. 5) through a standard least squares method using the value of $\mathrm{K}$ calculated in the previous step and obtaining the values of $\mathrm{d}$ and $\beta$. The computed values were $\mathrm{d}=2.5 \times 10^{-5}( \pm 5 \%)$ and $\beta=0 \mathrm{~m}^{-1}$ for the granite fracture, and $\mathrm{d}=2.2 \times 10^{-5}( \pm 2 \%)$ and $\beta=1.5( \pm 2 \%) \mathrm{m}^{-1}$ for the granite fracture. Percentages represent $\pm 2 \sigma$, with $\sigma$ being an estimate of the relative standard deviation of the measurements (95\% confidence interval).

\subsection{Equivalent and shear viscosity relations}

Eq. (30) was numerically solved within the involved range of $u$ for both fractures using an implicit Runge-Kutta method. From (23), it can be deduced that $\alpha$ becomes the constant value $\frac{1}{\sqrt{3}}\left(2+\frac{1}{n}\right)$ for Herschel-Bulkley shear-thinning fluids $(0<\mathrm{n}<1)$ flowing at very high 
585 values of $u$, i.e. when $u \gg \frac{\tau_{0} h^{n}}{2^{n} 3^{n / 2} \alpha^{n}}$. Given that the shift parameter is known to be greater than 586 unity [Chauveteau, 1982; Sorbie et al., 1989; López, 2003; Comba et al., 2011], the 587 preceding condition will be respected if $\mathrm{u} \gg \mathrm{u}^{*}=\frac{\tau_{0} \mathrm{~h}^{\mathrm{n}}}{2^{\mathrm{n}_{3}} \mathrm{n}^{\mathrm{n}}}$. Consequently, the boundary 588 condition $\alpha\left(\mathrm{u}=10^{5} \mathrm{u}^{*}\right)=\frac{1}{\sqrt{3}}\left(2+\frac{1}{\mathrm{n}}\right)$ was used to numerically solve Eq. (30). The resulting $\alpha$ 589 versus $u$ functions are presented in Figure 2(b) and 1(d).

591 Analogously, Eq. (29) was numerically solved within the range of u used by Rodríguez de 592 Castro and Radilla [2016a] for both fractures. From (22), it can be deduced that $\alpha$ becomes 593 the constant value $\sqrt{3}$ for Carreau shear-thinning fluids $(0<\mathrm{n}<1)$ flowing at very high 594 values of $u$, i.e. when $u \gg\left[\frac{2^{n} \alpha^{n-1} h^{1-n}}{6 \mu_{\infty}} \operatorname{Max}\left(3^{\frac{n-1}{2}}, 3^{\frac{n+1}{2}} c\right)\right]^{\frac{1}{1-n}}$. Since the shift parameter is 595 known to be greater than unity, the preceding condition will be respected if $u \gg$ $596 \mathrm{u}^{*}=\left[\frac{2^{\mathrm{n}} \mathrm{h}^{1-\mathrm{n}}}{6 \mu_{\infty}} \operatorname{Max}\left(3^{\frac{\mathrm{n}-1}{2}}, 3^{\frac{\mathrm{n}+1}{2}} \mathrm{c}\right)\right]^{\frac{1}{1-\mathrm{n}}}$. Therefore, the boundary condition $\alpha\left(\mathrm{u}=10^{5} \mathrm{u}^{*}\right)=\sqrt{3}$ was used to numerically solve Eq. (29). The resulting $\alpha$ versus u functions are presented in 598 Figure 2(a) and 1(c).

It can be noted that the relation between $\alpha$ and $\mathrm{u}$ strongly depends on polymer concentration as shown in Figure 2. Indeed, the dependence of $\alpha$ on $\mathrm{u}$ is weaker for the low polymer concentration as expected from their less pronounced shear-thinning behaviour. It is also remarked that this dependence of $\alpha$ on $\mathrm{u}$ is less significant as $\mathrm{u}$ increases and $\alpha$ approaches the limit value $\lim _{u \rightarrow \infty} \alpha(u)$. This implies that assuming a constant value of $\alpha$ should lead to acceptable levels of accuracy in the prediction of the $\nabla \mathrm{P}-\mathrm{u}$ relations within the high-u region. 

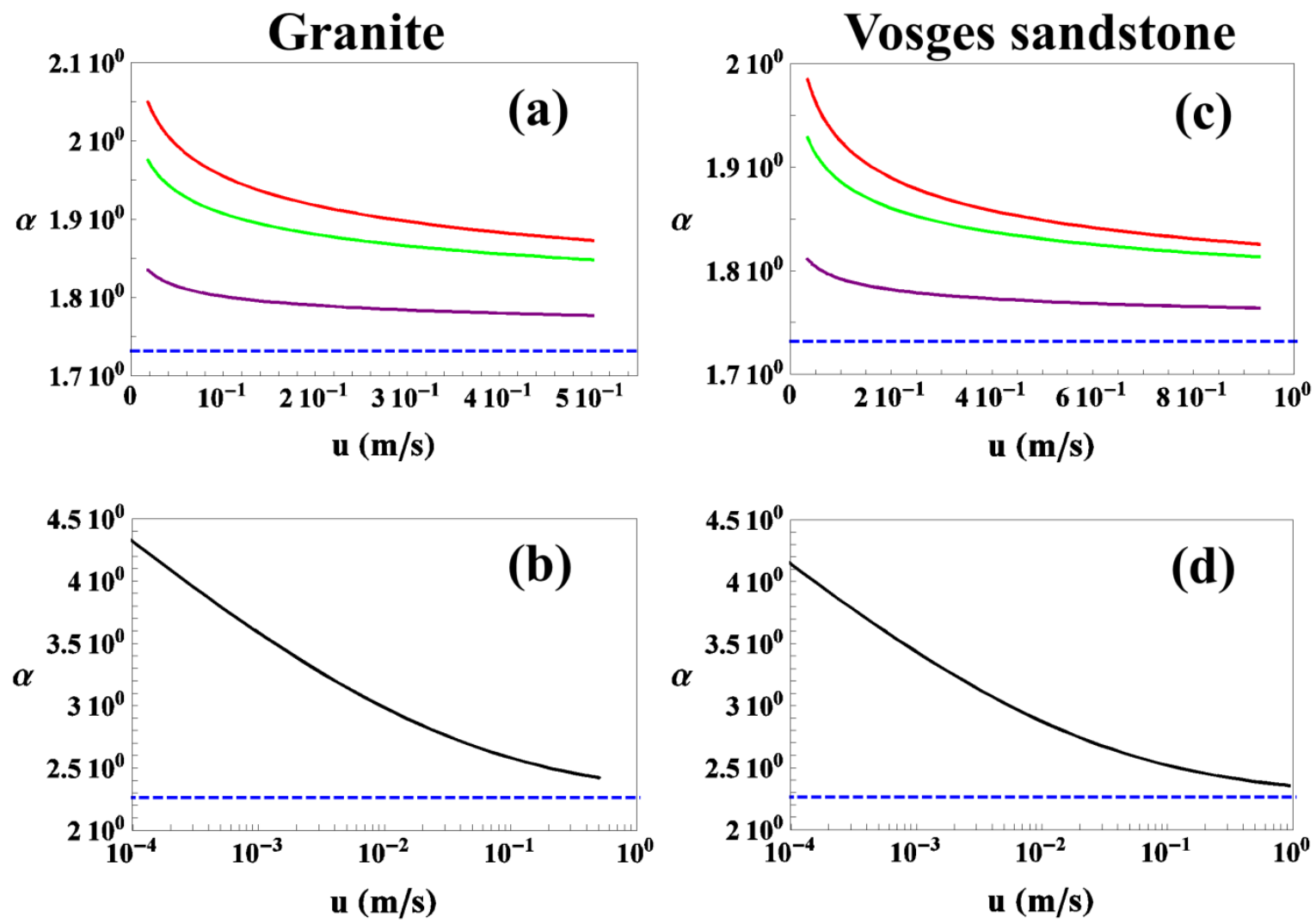

607 $\mathbf{u}(\mathbf{m} / \mathbf{s})$

608

609

610

611

612

613

614

615

616

617

618

Figure 2. $\alpha(\mathrm{u})$ functions as numerically obtained from Eqs. (29) and (30). (a,c) correspond to the Carreau fluids used by Rodríguez de Castro and Radilla [2016a]. (b,d) correspond to the 7000 ppm solution used in the present experiments. Solid lines represent the computed $\alpha(\mathrm{u})$ functions and dashed lines represent $\lim _{\mathrm{u} \rightarrow \infty} \alpha(\mathrm{u})$. Purple lines correspond to the $200 \mathrm{ppm}$ Carreau fluid, green lines to the 500 ppm Carreau fluid, red lines to the $700 \mathrm{ppm}$ Carreau fluid and black lines to the 7000 ppm yield stress fluid.

$\mu_{\mathrm{pm}, \mathrm{ysf}}$ was computed for the flow of the $7000 \mathrm{ppm}$ solution through each fracture using Eq.

(13). Two different approaches were followed: 1$)$ the constant value $\alpha=\frac{1}{\sqrt{3}}\left(2+\frac{1}{n}\right)$ was used in Eq. (13) and 2) the $\alpha(\mathrm{u})$ function obtained as explained above was used in Eq. (13). The results of both approaches are presented in Figure 3, together with $\mu_{\mathrm{eq}}$ as obtained with Eq. 
619 (11) from the $\nabla \mathrm{P}-\mathrm{u}$ measurements. In this figure, it can be observed that $\mu_{\mathrm{eq}}$ is close to $620 \mu_{\mathrm{pm}, \mathrm{ysf}}$ at high values $\mathrm{u}$ for both the constant $\alpha$ and the variable- $\alpha$ methods. However, this is not the case at low and moderate values of $u$ for which $\mu_{\mathrm{eq}}$ approaches clearly better $\mu_{\mathrm{pm}, \mathrm{ysf}}$ 622 with the variable- $\alpha$ method. It should be highlighted that xanthan gum may induce a depleted 623 layer close to pore walls with a lesser concentration in that region. This produces an apparent 624 wall slip which leads to a reduced average viscosity in the pores, mainly at low values of $u$ [Chauveteau, 1982; Sorbie, 1991b]. However, in the case of the present fractures, the dimensions of the macromolecules is negligible with respect to the fracture apertures so this effect is not observed and $\mu_{\mathrm{eq}}$ is very close to $\mu_{\mathrm{pm}, \mathrm{ysf}}$ even at low values of $\mathrm{u}$. This shows that that the effect of fluid-solid interactions (e.g. polymer mechanical degradation and apparent wall slip) on the relationship between viscosity and shear rate is negligible [González et al., 2005; Amundarain et al., 2009; Rodríguez de Castro et al., 2016b]. Also, it is expected that $\mu_{\mathrm{eq}}>\mu_{\mathrm{pm}, \mathrm{ysf}}$ at high values of $\mathrm{u}$ in the presence of important inertial effects [Tosco et al., 2013; Rodríguez de Castro and Radilla, 2016a]. The fact that no important deviation of $\mu_{\mathrm{eq}}$ with respect to $\mu_{\mathrm{pm}, \mathrm{ysf}}$ is observed in the present experiments reflects that inertial effects are not significant. Moreover, Figure 3 shows that the shear rates involved in the flow through the Vosges sandstone fracture are higher than those involved in the flow through the granite fracture. This is coherent with the highest values of $u$ and the lowest permeability of the Vosges sandstone fracture. 


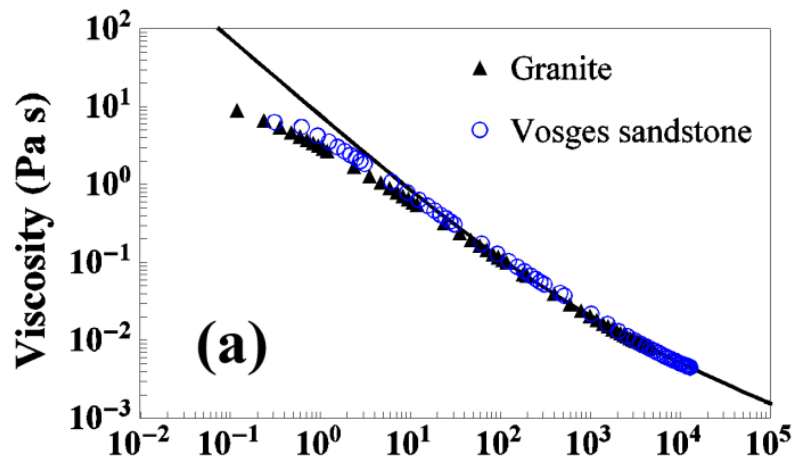

Shear rate $\left(s^{-1}\right)$

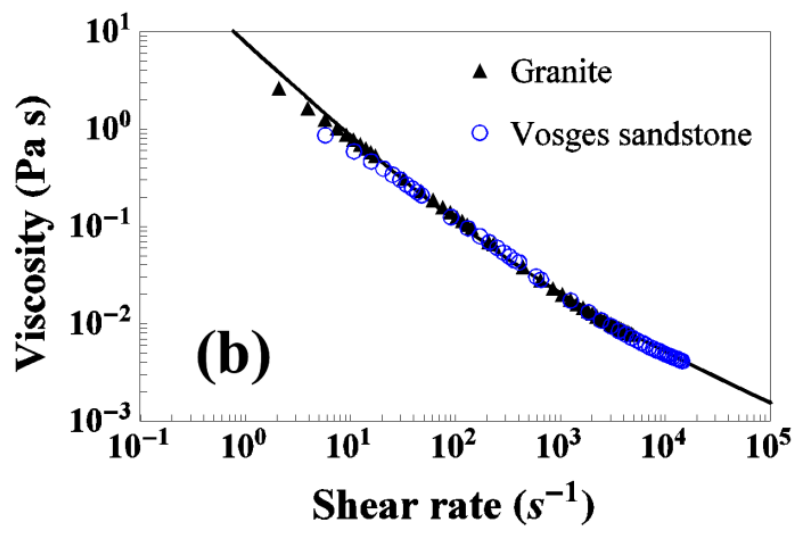

641 Figure 3. $\mu_{\mathrm{eq}}$ and $\mu_{\mathrm{pm}, \mathrm{ysf}}$ for the yield stress fluids used in the present experiments. Symbols

642 represent $\mu_{\mathrm{eq}}$ and solid lines represent $\mu_{\mathrm{pm}}$. (a) Corresponds to $\alpha=\frac{1}{\sqrt{3}}\left(2+\frac{1}{\mathrm{n}}\right)$.

643 Correspond to the $\alpha(\mathrm{u})$ functions presented in Figure 2.

It should be noted that the two-parameter power law model used in most of the preceding works dealing with shear-thinning fluids [Chhabra and Srinivas, 1991; Rao and Chhabra, 1993; Sabiri and Comiti, 1994; Smit and du Plessis, 1997; Tiu et al. 1997; Machac et al., 1998; Chhabra et al., 2001; Broniarz-Press et al., 2007] is not appropriate to study nonDarcian flow as the involved shear rates are high and close to the upper Newtonian plateau of viscosity [Woudberg et al., 2006; Fayed et al., 2016], which is not taken into account by this model. In contrast, the empirical Carreau model [Carreau, 1972] can accurately predict the variation in the viscosity at all shear rates and is known to successfully represent the shear- 
thinning behaviour of xanthan gum semi-dilute solutions [Sorbie et al., 1989; López et al., 2003; Rodríguez de Castro et al., 2016b; Rodríguez de Castro and Radilla, 2016a]. Although Herschel-Bulkley model does not include an upper Newtonian plateau viscosity, there is less concern in the case of this type of fluids. Indeed, as can be observed in figures 3 and $S_{3}$, the high levels of viscosity presented by the concentrated solutions injected in the present experiments are far from the upper plateau in all cases. This is in contrast with the results of Rodríguez de Castro and Radilla [2016a] for less concentrated xanthan gum solutions.

\subsection{Effects of yield stress on Reynolds number}

In previous works, it was shown that Reynolds number is not directly proportional to $\mathrm{u}$ for shear-thinning fluids, in contrast to the Newtonian case [Rodríguez de Castro and Radilla, 2016a, 2016b]. Indeed, according to Eqs. (13) and (14), an increase in u implies a decrease in viscosity which implies in turn an extra increase in Reynolds number. In this work, the effect of yield stress on the Re-u relationship was also analysed. To do so, the Reynolds numbers obtained for the imposed values of u were calculated through Eq. (4) in the case of the granite fracture and Eq. (6) in the case of the Vosges sandstone fracture. $\mu_{\mathrm{pm}}$ was used for the calculation of Reynolds number. It is highlighted that $\mu_{\mathrm{pm}}$ accounts only for viscous effects and is consistent with the definition of Reynolds number as the ratio of inertial to viscous forces, in contrast to $\mu_{\mathrm{eq}}$ that accounts also for inertial effects. The results are presented in Figure 4. In this figure, it can be observed that Reynolds number is close to zero at low values of $u$ for the flow of the yield stress fluid in both fractures. 

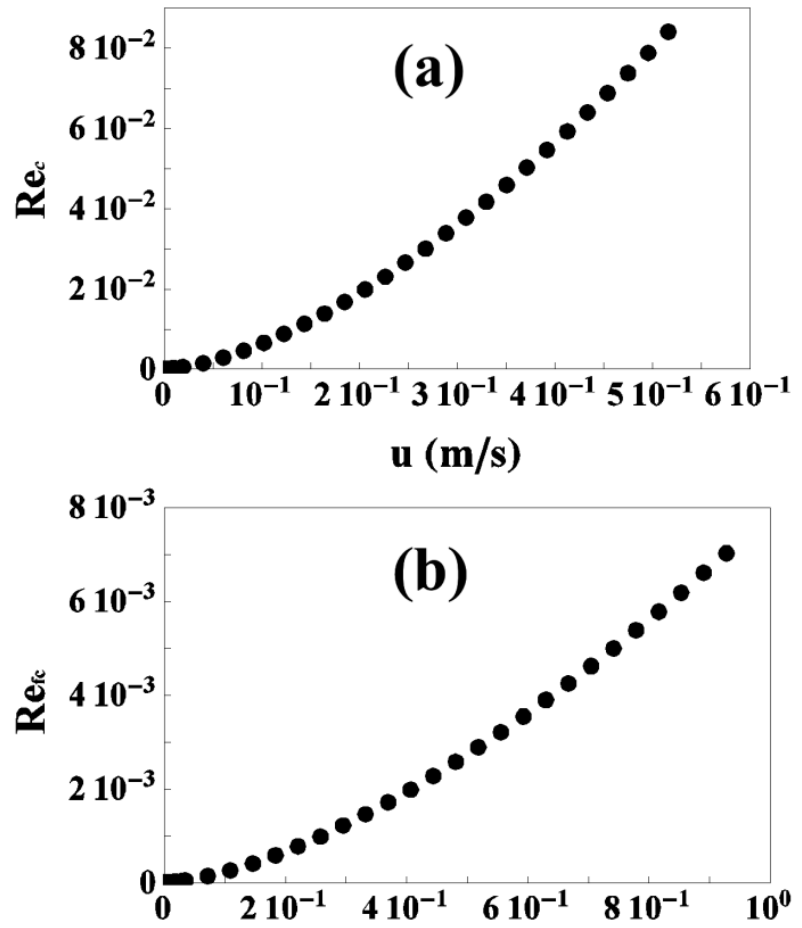

Figure 4. (a) $\mathrm{Re}_{\mathrm{c}}$ vs. $\mathrm{u}$ for the granite fracture (b) $\mathrm{Re}_{\mathrm{fc}} \mathrm{vs}$. $\mathrm{u}$ for the Vosges sandstone fracture.

679 From Figure 4, one can also deduce that the non-linear dependence of $\operatorname{Re}$ on u previously 680 reported for Carreau fluids is also observed for shear-thinning yield stress fluids. 681 Furthermore, as reflected in the same figure, there is a threshold value in terms of $u$ below which Re is very close to zero for the injection of yield stress fluids. This threshold value arises from the yield stress of the fluid. In fact, for a yield stress fluid, viscosity approaches infinity at low shear rates leading to very low values of Re. Also, the critical value of Re for the transition to non-Darcian regime was reported to be close to $\mathrm{Re}_{\mathrm{c}}=0.3$ for the granite fracture and $\mathrm{Re}_{\mathrm{fc}}=0.05$ for the Vosges sandstone fracture [Rodríguez de Castro and Radilla, 2016a]. As can be seen in Figure 4, the Re obtained for the present experiments are lower than these critical values in both fractures, so no important inertial effects are expected. The

689 ratio between inertial and viscous pressure losses was calculated from Eq. (33) as $\frac{\Delta \mathrm{P}_{\text {inertial }}}{\Delta \mathrm{P}_{\text {viscous }}}=$ 
$690 \frac{\beta \rho u+\frac{\gamma \rho^{2}}{\mu \mathrm{pm}} \mathrm{u}^{2}}{\frac{12 \mu \mathrm{pm}}{\mathrm{h}^{2}}}$ leading to values of $\frac{\Delta \mathrm{P}_{\text {inertial }}}{\Delta \mathrm{P}_{\text {viscous }}}<7.1 \times 10^{-3}$ for the granite fracture and $\frac{\Delta \mathrm{P}_{\text {inertial }}}{\Delta \mathrm{P}_{\text {viscous }}}<3.1$

$691 \times 10^{-2}$ for the Vosges sandstone fracture. This confirms that inertial pressure losses are not 692 relatively important, in contrast to the experiments with Carreau fluids performed by 693 [Rodríguez de Castro and Radilla, 2016a].

694

\subsection{Experimental validation of the proposed prediction methods}

Eq. (33) was used to predict the relation between $\nabla \mathrm{P}$ and $\mathrm{u}$ for the injection of the $7000 \mathrm{ppm}$ solution through the fractures. The $\beta$ and $d$ values in Eqs. (2-5) do not depend on polymer concentration as shown by Rodríguez de Castro and Radilla [2016a, 2016b], so the values obtained from water injection (subsection 4.1) were used. The obtained predictions are presented in Figure 5 together with the experimental results of measurements performed in the present work. In this figure, the errors bars correspond to a $95 \%$ confidence interval as explained in subsection 3.1. The results are presented in a log-log scale in order to allow visibility of all the range of measurements and in a linear scale so as to show that the form of the curves is the same as that of the rheogram of a yield stress fluid (Figure S3). From these results, the accuracy of the proposed methods for the prediction of $\nabla \mathrm{P}$ as a function of $\mathrm{u}$ during the flow of yield stress fluids through rough-walled fractures can be assessed. Figure 5 shows that the variable- $\alpha$ approach provides more accurate predictions within the low and moderate $\mathrm{u}$ regions, which is in agreement with the arguments presented above. However, a less important difference is obtained between both methods for the highest values of $\mathrm{u}$. It is observed that the variable- $\alpha$ method successfully predicts the $\nabla \mathrm{P}-\mathrm{u}$ relationship for the flow of the yield stress fluid through both fractures, even though the obtained predictions are slightly less accurate in the case of the Vosges sandstone. 

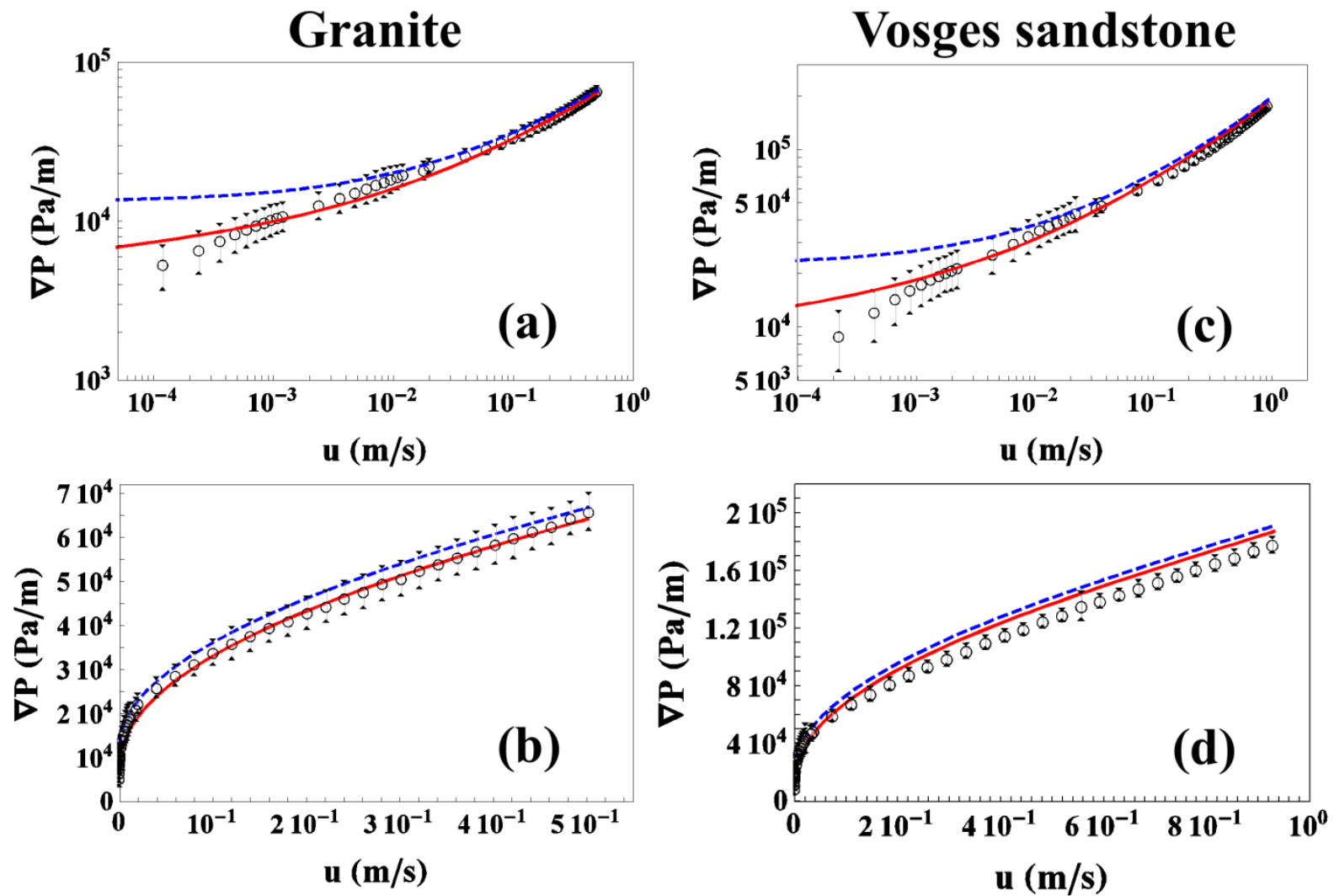

Figure 5. $\nabla \mathrm{P}$ as a function of $\mathrm{u}$ corresponding to $(\mathrm{a}, \mathrm{b})$ Granite and $(\mathrm{c}, \mathrm{d})$ Vosges sandstone fractures. Symbols represent experimental data, red solid lines represent predictions using Eq. (33) with the $\alpha(\mathrm{u})$ functions presented in Figure 2 and blue dashed lines represent predictions using Eq. (33) with $\alpha=\frac{1}{\sqrt{3}}\left(2+\frac{1}{n}\right)$.

With the objective of assessing the accuracy of the proposed predictions in the case of Carreau fluids, Eq. (33) was also used to predict the u-VP relations for the injection of the three Carreau fluids used by Rodríguez de Castro and Radilla [2016a], and the results were compared to their experimental data in Figure 6. The average deviations between predictions and experiments corresponding to the whole range of explored $\mathrm{u}$ for all tested fluids and fractures are included as supporting information of this article (Table S1). As can be seen in 
727 Figure 6, the predictions coming from both methods (constant- $\alpha$ and variable- $\alpha$ ) methods are 728 almost identical in all cases. This is explained by the proximity of all the $\alpha$ values to the limit 729 value $\sqrt{3}$ (Figure 2) within the range of imposed $u$. Furthermore, the low and moderate $u$ 730 regions were not explored by [Rodríguez de Castro and Radilla, 2016a], while it is precisely 731 in these regions where more important differences are expected between both approaches. 732 However, the predictions obtained for the covered range of $u$ is in very good agreement with 733 the experimental data, apart from the $500 \mathrm{ppm}$ - granite pair which will need further study. 734 Moreover, it is also remarked that Eq. (33) successfully takes into account the inertial effects, 735 which are important for the flow of the injected Carreau fluids at the involved values of $\mathrm{u}$. 

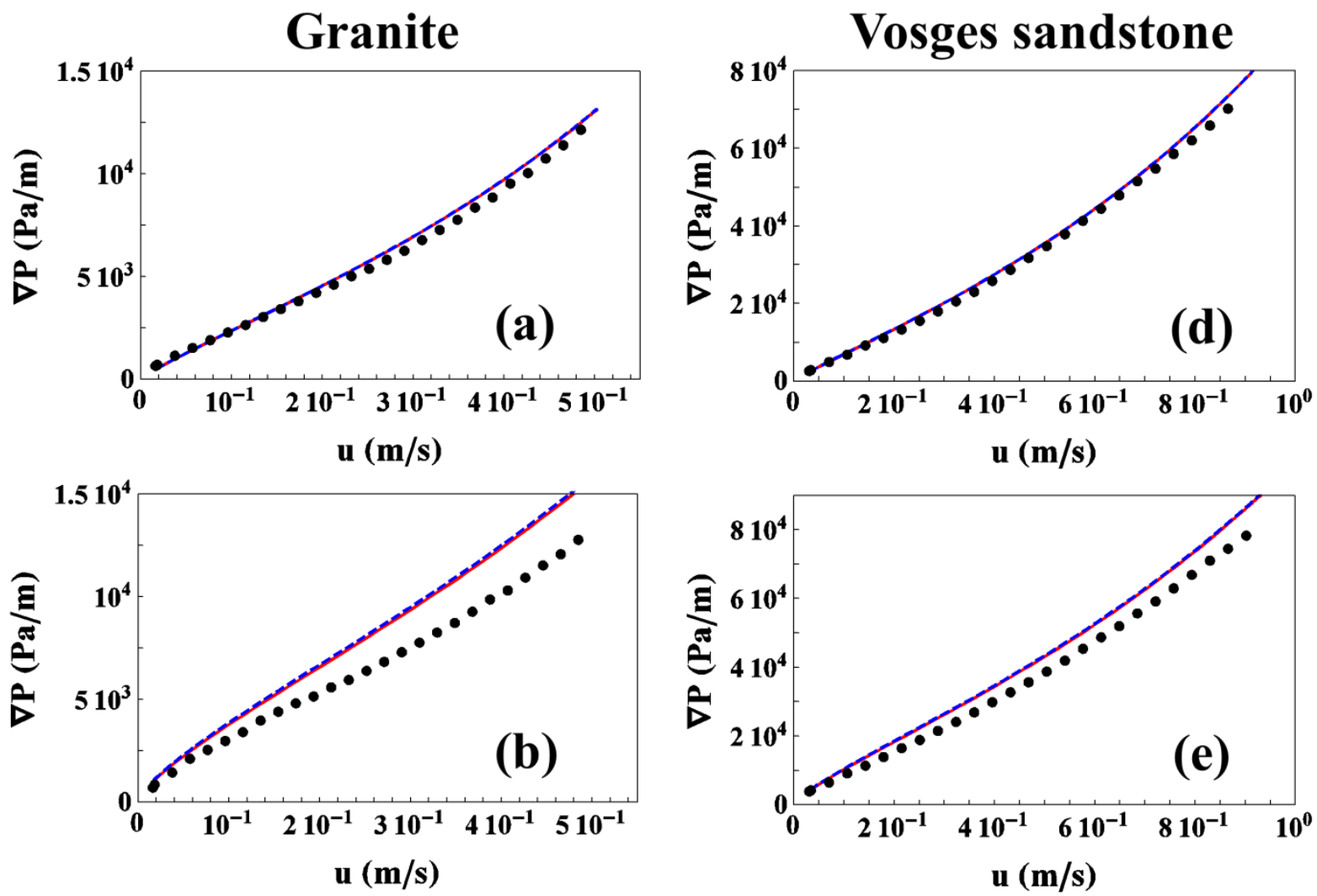

739
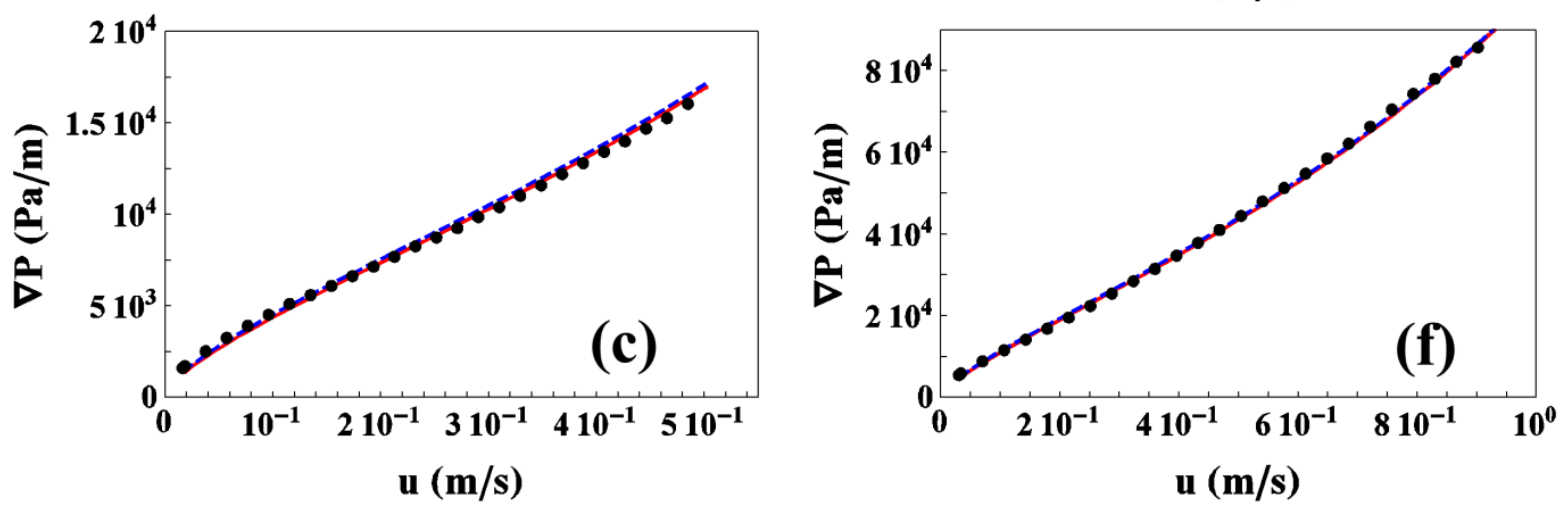

740 Figure 6. $\nabla \mathrm{P}$ as a function of $\mathrm{u}$ corresponding the injection of the three Carreau fluids used

741 by Rodríguez de Castro and Radilla [2016a] through (a,b,c) Granite and (d,e,f) Vosges

742 sandstone fractures. Symbols represent experimental data, red solid lines represent predictions using Eq. (33) with the $\alpha(\mathrm{u})$ functions presented in Figure 2 and blue dashed lines

744 represent predictions using Eq. (33) with $\alpha=\sqrt{3}$. 
749 As explained in subsection 3.2., the concentrated xanthan gum solutions used in our experiments present an apparent yield stress, so they should be referred to as pseudo-yield stress fluids. In this regard, Lipscomb and Denn [1984] showed that the classical lubrication approximation, which essentially assumes that flow is locally fully-developed, can be applied to this type of fluids, while it cannot be successfully applied to ideal yield stress fluids with a real yield stress. These authors argued that rigid plug regions should not exist in complex geometries according to classical lubrication. Indeed, this theory predicts that plug-velocity changes slowly as aperture varies, so the plug region cannot be truly unyielded for continuity reasons. The latter is known as lubrication paradox [Lipscomb and Denn, 1984; Frigaard and Ryan, 2004; Lavrov, 2013]. However, as showed by Lipscomb and Denn [1984], both real and pseudo-yield stress fluids may exhibit a near-plug-like region in a complex flow field. For fully developed flows, the depth of the plug as a function of $\nabla \mathrm{P}$ is given by [Lipscomb and Denn, 1984]:

$\mathrm{h}_{0}=\frac{2 \tau_{0}}{\nabla \mathrm{P}}$

As a first simple approach similar to the one presented by Auradou [2008], we will assume that the fracture space can be modelled as being a bundle of parallel rectangular canals of length $L$, width $w_{i}$ and aperture $h_{i}$, with $w_{i} \gg h_{i} . h_{i}$ is expected to vary along the flow paths. However, given the strong dependence of $\nabla \mathrm{P}$ on the canal aperture in the case of shearthinning fluids, we will also assume that the pressure drop along a percolating path is located 
exclusively in the section of smallest aperture $\mathrm{h}_{0}$. By doing so, it can be deduced that the

769

770

771

772

773

774

775

776

777

778

779

780

Figure 7. $h_{0}$ as a function of $u$ for the flow of the yield stress fluid through the (a) granite

fluid will flow through a percolating path only if the minimum local aperture is superior to $\mathrm{h}_{0}$ as given by Eq. (34). Therefore, the number of percolating pathways is expected to increase as $\nabla \mathrm{P}$ and $\mathrm{u}$ increase. $\mathrm{h}_{0}$ has been presented as a function of $\mathrm{u}$ for the present flow experiments with yield stress fluids in Figure 7. This figure shows that $h_{0}$ decreases with $u$, as expected. Indeed, only the pathways with the highest apertures participate in the flow at the lowest values of $\mathrm{u}$, while pathways with smaller values of $\mathrm{h}_{0}$ are progressively incorporated as u increases. According to this simple approach, the flow pathways including the minimum apertures of the fracture would not participate in the flow, even at the highest $\mathrm{u}$, so unyielded fluid regions would exist (in agreement with Frigaard and Ryan [2004]).
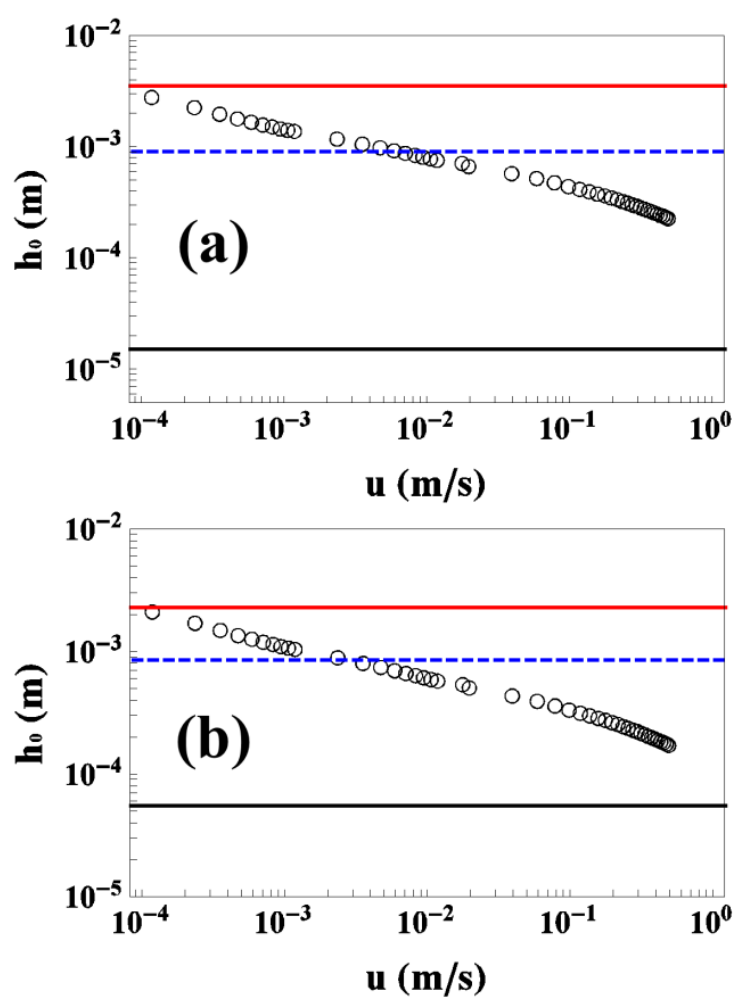

fracture and the (b) Vosges sandstone fracture. The experimental data are represented as void 
782

783

784

785

786

787

788

789

$$
\nabla \mathrm{P}=\frac{\mathrm{C}_{1}}{\alpha}+\mathrm{C}_{2} \alpha^{\mathrm{n}-1} \mathrm{u}^{\mathrm{n}}
$$

791 with $\mathrm{C}_{1}=\frac{2 \sqrt{3} \tau_{0}}{\mathrm{~h}}$ and $\mathrm{C}_{2}=\frac{2^{\mathrm{n}+1} 3^{\frac{\mathrm{n}+1}{2}} \mathrm{a}}{\mathrm{h}^{\mathrm{n}+1}}$

793 In the high flow rates region, i.e. when $u \gg \frac{\tau_{0} h^{n}}{2^{n} 3^{n / 2}}, \alpha$ can be considered a constant value $\alpha=$ $794 \frac{1}{\sqrt{3}}\left(2+\frac{1}{n}\right)$ and Eq. (31) leads to:

795

$$
\nabla \mathrm{P}=\nabla \mathrm{P}_{0}+\mathrm{Cu}^{\mathrm{n}}
$$


797 with $\nabla \mathrm{P}_{0}=\frac{6 \mathrm{n} \tau_{0}}{\mathrm{~h}(2 \mathrm{n}+1)}$ and $\mathrm{C}=\frac{6 \mathrm{an}\left(\frac{2+4 \mathrm{n}}{\mathrm{hn}}\right)^{\mathrm{n}}}{\mathrm{h}+2 \mathrm{hn}}$. This is in agreement with the results of Talon et al. 798 [2014], who stated that $u$ scales linearly as $\left(\nabla P-\nabla P_{0}\right)$ in the case of a Bingham fluid $(n=1)$ 799 flowing at high u through a one-dimensional channel. Also, Nash and Rees [2017] showed 800 that the manner in which flow begins once the threshold pressure gradient is exceeded 801 strongly depends on the channel size distribution of the porous media. The same authors [Talon et al., 2014; Nash and Rees, 2017] proved that $\nabla \mathrm{P}_{0}$ is higher than the actual threshold pressure, which is consistent with our results given that $\alpha$ increases as $u$ tends to zero (Figure 2). Roustaei et al. [2016] numerically showed that unyielded plug regions appear close to the fracture wall and in the deeper layers (fouling layers) when injecting yield stress fluids in short fractures, especially at low values of $\mathrm{u}$. These researchers showed that Darcy-type flow laws are limited to $\mathrm{H} / \mathrm{L} \ll 1, \mathrm{H}$ being a half of the difference between the maximum and the minimum aperture of the fractures. In the case of the granite sandstone used in the present work $\mathrm{H} / \mathrm{L}=6.2 \times 10^{-3}$ while $\mathrm{H} / \mathrm{L}=5.5 \times 10^{-3}$ for the granite sandstone as shown in supporting figures, so a Darcy-type approach is expected to be valid.

Lavrov [2015] developed analytical solutions for the flow of truncated power law fluids through smooth-walled fractures. Truncated power-law fluids, unlike Carreau fluids, enable a closed-form solution for the flow between plane parallel walls while exhibiting more realistic behaviour than simple power-law fluids for commonly used polymer solutions. However, truncated power-law fails to model the real behaviour of these complex fluids at shear rates lying within the transition region between the shear-thinning region and the upper Newtonian plateau. Therefore, this model is not expected to provide accurate predictions in the wide range of shear-rates explored in the present experiments. 
821 One may wonder whether the proposed procedure is simpler than performing a numerical

822 solution to the actual flow equations, without invoking a bundle-of-capillaries approximation.

823 In this sense, it should be highlighted that performing a numerical solution to the actual flow

824 equations would imply using the size distribution of the flow paths as an input for the model.

825 This information on the size distribution of the flow paths is rarely available in real

826 applications, while the average aperture of the fracture can be more easily estimated or

827 measured from water flow experiments. It is reminded that the objective of this work is to

828 present a simple method to predict the pressure drop for the flow of shear-thinning fluids

829 through tough-walled rock fractures. Therefore, using hardly accessible inputs as needed to 830 perform a numerical solution to the actual flow equations is not a valid approach.

832 Also, it is noted that in our experiments with yield stress fluids, the total pressure drop through the fractures was successfully predicted from the values of $K, \gamma$ and $\beta$ obtained from water injection without any significant deviation. Therefore, elongational viscosity effects have been shown to be negligible in the case of the present experiments with yield stress

836 fluids as they were with the Carreau fluids used by [Rodríguez de Castro and Radilla, 837 2016a]. 


\section{Summary and conclusions}

840 A simple method to extend Darcy's law, weak inertia cubic law and full cubic law to the flow 841 of yield stress fluids and Carreau fluids in rough-walled natural fractures has been presented 842 in the present work. In this method, the values of the shift parameter $\alpha$ between the $\mu_{\mathrm{pm}}$ 843 measured in the rheometer and the $\mu_{\mathrm{eq}}$ observed during the flow in the porous media is predicted through identification of the apparent shear rate with the maximum wall shear rate in a section with aperture $h$. The inputs of the method are only the shear rheology parameters of the fluid, the hydraulic aperture of the fracture and the inertial coefficients $\gamma$ and $\beta$. On the basis of our results, an efficient protocol to predict $\nabla \mathrm{P}$ as a function of $\mathrm{u}$ is proposed here:

1) Determine the shear-rheology parameters of the fluid: $\left(\tau_{0}, a, n\right)$ for Herschel-Bulkley fluids or $\left(\mu_{\infty}, \mathrm{c}, \mathrm{n}\right)$ for Carreau fluids.

2) Mesure h, $\beta$ and $\gamma$ from Newtonian-flow experiments. Alternatively, h can be deduced equation (29) or (30) to obtain $\alpha(u)$.

3.2) When only high values of $u$ are involved $\left(u \gg \frac{\tau_{0} h^{n}}{2^{n} 3^{n / 2}}\right.$ for yield stress fluids or $\mathrm{u} \gg\left[\frac{2^{\mathrm{n}} \mathrm{h}^{1-\mathrm{n}}}{6 \mu_{\infty}} \operatorname{Max}\left(3^{\frac{\mathrm{n}-1}{2}}, 3^{\frac{\mathrm{n}+1}{2}} \mathrm{c}\right)\right]^{\frac{1}{1-\mathrm{n}}}$ for Carreau fluids), use a constant value $\alpha=\frac{1}{\sqrt{3}}\left(2+\frac{1}{n}\right)$ for Hershel-Bulkley fluids or $\alpha=\sqrt{3}$ for Carreau fluids.

4) Use Eq. (13) or (14) to calculate $\mu_{\mathrm{pm}, \text { Carreau }}$ or $\mu_{\mathrm{pm}, \mathrm{ysf}}$ 
864 Flow experiments of yield stress fluids covering a wide range of $u(\sim 3.6$ orders of

5) Use Eq. (33) to calculate $\nabla P$ as a function of $u$, with $\gamma=0$ in the case of Forchheimer's law (strong inertia regime), $\beta=0$ in the case of a cubic law (weak inertia regime), $\beta=0$ and $\gamma=0$ in the case of Darcy's law (creeping flow). magnitude) have been performed and compared with the predictions of the proposed method, showing good agreement. It has been observed that the existence of a yield stress reduces significantly the value of Reynolds, so the inertial effects are negligible within the explored range of u. Consequently, Darcy's law provide accurate u- $\nabla \mathrm{P}$ predictions in contrast to the case of less concentrated solutions with no yield stress [Rodríguez de Castro and Radilla, 2016a]. Also, the experimental results obtained in the non-Darcian shear-thinning flow experiments through rough-walled fractures conducted by [Rodríguez de Castro and Radilla, 2016a] have been compared with the predictions of the proposed method, showing good agreement also in the case of Carreau fluids. It should be noted that good predictions of the pressure drop-flow rate relations are obtained by only using the global parameters h, $\beta$ and $\gamma$ as inputs. Therefore, no significant effects of the aperture distributions of the fractures have been observed.

The variable- $\alpha$ approach leads to a very good overlap between $\mu_{\mathrm{pm}}$ and the $\mu_{\mathrm{eq}}$ over the wide range of $\mathrm{u}$ investigated in this work. Our results can be included in computational studies of large-scale nonlinear flow in fractured rocks, as suggested in the works of Javadi et al. [2014]. These conclusions must now be extended to other types of rough-walled rock fractures. 


\section{Acknowledgments}

884

885 The authors would like to thank Frédéric Bastien for his assistance with the experimental 886 setup.

887

888

Supporting data are included as four figures and a table in SI files; any additional data may be 889 obtained from the authors (antonio.rodriguezdecastro@ensam.eu).

890

891

References

892

893 Agnaou, M., D. Lasseux, and A. Ahmadi (2016), From steady to unsteady laminar flow in 894 model porous structures : an investigation of the first Hopf bifurcation, Computers and Fluids $895136,67-82$.

896

897 Al-Fariss, T., and K. L. Pinder (1987), Flow through porous media of a shear-thinning liquid 898 with yield stress, Can. J. CHem. Eng. 65, 391-405.

899

900 Ambari, A., M. Benhamou, S. Roux, and E. Guyon (1990): Distribution des tailles des pores 901 d'un milieu poreux déterminée par l'écoulement d'un fluide à seuil. C. R. Acad. Sci. Paris t.

902 311(11), 1291-1295

903 
Sáez (2009), Solutions of xanthan gum/guar gum mixtures: shear rheology, porous media flow, and solids transport in annular flow, Rheologica Acta 48, 491-498.

907

Auradou, H., A. Boschan, R. Chertcoff, S. Gabbanelli, J. P. Hulin, and I. Ippolito (2008),

Enhancement of velocity contrasts by shear-thinning solutions flowing in a rough fracture, $J$.

Non-Newtonian Fluid Mech. 153, 53-61.

911

Ball, J. T., and M. J. Pitts (1984), Effect of Varying Polyacrylamide Molecular Weight on

Tertiary Oil Recovery From Porous Media of Varying Permeability, SPE Enhanced Oil Recovery Symposium, 15-18 April, Tulsa, Oklahoma.

915

Benmouffok-Benbelkacem, G., F. Caton, C. Baravian, and S. Skali-Lami (2010), Non-linear viscoelasticity and temporal behavior of typical yield stress fluids. Carbopol, Xanthan and Ketchup, Rheol. Acta, 49, 305-314.

Boronin, S. A., A. A. Osiptsov, and J. Desroches (2015), Displacement of yield-stress fluids in a fracture, International Journal of Multiphase Flow, 76, 47-63.

Broniarz-Press, L., P. Agacinski, and J. Rozanski (2007), Shear-thinning fluids flow in fixed and fluidized beds, International Journal of Multiphase Flow, 33, 675-689. 
929 Brush, D.J., and N. R. Thomson (2003), Fluid flow in synthetic rough walled fractures:

930 Navier-Stokes, Stokes, and local cubic law simulations, Water Resour. Res. 39 (4), 1085.

931 doi:10.1029/2002WR001346

932

933 Buès, M., M. Panfilov, and C. Oltean (2004), Macroscale model and inertia-viscous effects 934 for Navier-Stokes flow in a radial fracture with corrugated walls, J. Fluid Mech. 504, 41-60. doi:10.1017/S002211200400816X

936

937 Carnali, J.O. (1991), A dispersed anisotropic phase as the origin of the weak-gel properties of 938 aqueous xanthan gum, J. Appl. Polym. Sci., 43(5), 929 - 941

939

940 Carreau, P.J. (1972), Rheological equations from molecular network theories, Trans. Soc. 941 Rheol., 16,99-127.

Chase, G. G., and P. Dachavijit (2005), A correlation for yield stress fluid flow through 944 packed beds, Rheol. Acta, 44, 495 - 501.

945

946 Chauveteau, G. (1982), Rodlike Polymer Solution Flow through Fine Pores: Influence of 947 Pore Size on Rheological Behavior, J. Rheol., 26, 111.

949 Chauveteau, G., and C. Thirriot (1967), Régimes d'écoulement en milieu poreux et limite de 950 la loi de Darcy, La Houille Blanche, 2, 141-148. doi:10.1051/lhb/1967009 
952 Chauveteau, G., and A. Zaitoun (Sept. 1981), Basic Rheological Behavior of Xanthan 953 Polysaccharide Solutions in Porous Media : Effects of Pore Size and Polymer Concentration, 954 European Symposium on Enhanced Oil Recovery, Bournemouth, England.

955

956 Chen, Z., S. L. Lyons, and G. Qin (2001), Derivation of the Forchheimer law via 957 homogenization, Transp. Porous Media 44 (2), 325-335. doi:10.1023/A:1010749114251

958

959

Chen, M., W. Rossen, and Y. C. Yortsos (2005), The flow and displacement in porous media 960 of fluids with yield stress, Chem. Eng. Sci., 60, 4183-4202.

961

962

Chevalier, T., C. Chevalier, X. Clain, J. C. Dupla, J. Canou, S. Rodts, and P. Coussot (2013), 963

Darcy's law for yield stress fluid flowing through a porous medium, Journal of Non964 Newtonian Fluid Mechanics, 195, 57 - 66.

965

966

Chevalier, T., S. Rodts, X. Chateau, C. Chevalier, and P. Coussot (2014), Breaking of non967 Newtonian character in flows through a porous medium, Phys Rev E, 89, 023002.

968

969

970

Chhabra, R.P., J. Comiti, and I. Machac (2001), Flow of non-Newtonian fluids in fixed and fluidised beds, Chemical Engineering Science, 56, 1 - 27.

971

972 Chhabra, R.P., and B. K. Srinivas (1991), Non-Newtonian (purely viscous) fluid flow 973 through packed beads: effect of particle shape, Powder Technology, 67, 15 - 19. 
975 Comba, S., D. Dalmazzo, E. Santagata, and R. Sethi (2011), Rheological characterization of 976 xanthan suspensions of nanoscale iron for injection in porous media, Journal of Hazardous 977 Materials, 185, 598-605.

978

979 Cornell, D., and D.L Katz (1953), Flow of gases through consolidated porous media, Ind. 980 Eng. Chem., 45 (10), 2145-2153. doi:10.1021/ie50526a021

981

982 Coussot, P. (2005), Rheometry of Pastes, Suspensions, and Granular Materials, Applications 983 in Industry and Environment, Wiley.

984

985 986

Coussot, P. (2014), Yield stress fluid flows: A review of experimental data, Journal of NonNewtonian Fluid Mechanics, 211, 31-49.

987

988

Cvetkovic, V.D. (1986), A continuum approach to high velocity flow in a porous medium, 989 Transp. Porous Media, 1 (1), 63-97. doi:10.1007/BF01036526

990

991

992

Di Federico, V. (1997), Estimates of Equivalent Aperture for Non-Newtonian Flow in a Rough-walled Fracture, Int. J. Rock Mech. Min. Sci., 34(7), 1133-1137.

993

994 Di Federico, V., (1998). Non-Newtonian flow in a variable aperture fracture, Transport in 995 Porous Media, 30 (1), 75-86.

996

997 Di Federico, V. (2001), On non-Newtonian fluid flow in rough fractures, Water Resources 998 Research, 37(9), 2425-2430. 
1000 Dimitriou, C. J., and G. H. McKinley (2014), A comprehensive constitutive law for waxy 1001 crude oil: a thixotropic yield stress fluid, Soft Matter, 10, 6619-6644, 1002 http://dx.doi.org/10.1039/C4SM00578C

1003

1004

1005 consolidated isotropic porous media, Trans. Porous Med., 3, 145-161.

1006

1007

1008 and reconciliation of Forchheimer and Ergun relations, Exp. Therm. Fluid Sci., 57, 425-433.

1009

doi:10.1016/j.expthermflusci.2014.06.011.

1010

1011

1012

periodically nonuniform capillary tube, AIChE J., 19, 222-229. doi:10.1002/aic.690190204.

1013

1014

Economides M.J., and K. G. Nolte, 2000. Reservoir Stimulation. Third edition. Wiley, New 1015

York. El-Khatib, N. 2005. Immiscible Displacement of Non-Newtonian Fluids in Stratified 1016

Reservoirs, SPE Middle East Oil \& Gas Show and Conference, Bahrain. SPE 93394.

1017

1018

Fayed, H.E., N. A. Sheikh, and O. Iliev, 2016. On Laminar Flow of Non-Newtonian Fluids in

1019

Porous Media. Transport in Porous Media, 111, 253-264.

1020

1021

Firdaouss, M., J.-L. Guermond, and P. Le-quéré (1997), Nonlinear correction to Darcy’s law at low Reynolds numbers, J. Fluid Mech., 343, 331-350. doi:10.1017/S0022112097005843 
1024 Forchheimer, P. (1901), Wasserberwegng durch Boden. Forschtlft ver. D. Ing., 45(50), 178210251788

1026

1027 Fourar, M., G. Radilla, R. Lenormand, and C. Moyne (2004), On the non-linear behavior of a 1028 laminar single-phase flow through two and three-dimensional porous media, Adv. Water 1029 Resour. 27(6), 669-677. doi:10.1016/j.advwatres.2004.02.021

1030

1031 Frigaard, I. A., and D. P. Ryan, (2004), Flow of a visco-plastic fluid in a channel of slowly 1032 varying width, Journal of Non-Newtonian Fluid Mechanics, 123, 67-83.

1033

1034

Garcia-Ochoa, F., V.E. Santosa, J.A Casasb, and E. Gómez (2000), Xanthan gum: 1035 production, recovery, and properties, Biotechnol. Adv., 18, 549-579.

1036

1037 Geertsma, M. (1974), Estimating the coefficient of inertial resistance fluid flow through 1038 porous media, SPE J., 14 (5), 445-450. doi:10.2118/4706-PA

1039

1040 Giorgi, T. (1997), Derivation of the Forchheimer law via matched asymptotic expansions, 1041 Transp. Porous Media, 29 (2), 191-206. doi:10.1023/A:1006533931383

1043 González, J. M., A. J. Müller, M. F. Torres, and A. E. Sáez (2005), The role of shear and 1044 elongation in the flow of solutions of semi-flexible polymers through porous media, Rheol. Acta, 44, 396-405. doi:10.1007/s00397-004-0421-4 
1047 Hernández-Espriú, A., E. Sánchez-León, P. Martínez-Santos, and L. G. Torres (2013),

1048 Remediation of a diesel-contaminated soil from a pipeline accidental spill: enhanced 1049 biodegradation and soil washing processes using natural gums and surfactants, J. Soils 1050 Sediments, 13, $152-165$.

1051

1052

1053

Herschel, W. H., and R. Bulkley (1926), Konsistenzmessungen von Gummi-Benzollösungen, Kolloid-Zeitschrift, 39: 291. doi:10.1007/BF01432034

1054

1055

Hubbert, M. K. (1956), Darcy law and the field equations of the flow of underground fluids, 1056 Trans. Am. Inst. Min. Mandal. Eng., 207, 222 - 239.

1057

1058

1059

1060 4), 267-282. doi: 10.1016/ S0012-821X(01)00424-1

1061

1062

Javadi, M., M. Sharifzadeh, S. Kourosh, and M. Yasuhiro (2014), Critical Reynolds number

1063 for nonlinear flow through rough-walled fractures: The role of shear processes, Water Resour. Res., 50, 1789-1804. doi:10.1002/2013WR014610.

Jones, D.M., and K. Walters (1989), The behavior of polymer solutions in extension1067 dominated flows with applications to enhanced oil recovery, Rheol. Acta, 28, 482-498

1068

1069

Khodja, M. (2008), Les fluides de forage: étude des performances et considerations environnementales, $\mathrm{PhD}$ thesis, Institut National Polytechnique de Toulouse. 
1072 Lavrov, A. (2013), Non-Newtonian fluid flow in rough-walled fractures: A brief review, 1073 Proceedings of ISRM SINOROCK 2013, 18-20 June, Shanghai, China.

1074

1075 Lavrov, A. (2015), Flow of truncated power-law fluid between parallel walls for hydraulic 1076 fracturing applications, Journal of Non-Newtonian Fluid Mechanics, 223, 141-146.

1077

Lake, LW. (1989), Enhanced oil recovery, Englewood Cliffs, NJ: Prentice-Hall Inc.

1079

1080

Lipscomb, G. G., and M. M. Denn (1984), Flow of Bingham fluids in complex geometries, 1081 Journal of Non-Newtonian Fluid Mechanics, 14, 337-346.

1082

1083

López X., P. H. Valvatne, and M. J. Blunt (2003), Predictive network modeling of single1084 phase non-Newtonian flow in porous media, J. Colloid Interface Sci., 264(1), 256-265. 1085 doi:10.1016/s0021-9797(03)00310-2

1086

1087 López, X. (2004), PhD Thesis, Department of Earth Science \& Engineering Petroleum 1088 Engineering \& Rock Mechanics Group, Imperial College, London.

1089

1090

MacDonald, I. F., M. S. El-Sayed, K. Mow, and F. A. L. Dullien (1979), Flow through 1091 porous media, the Ergun equation revisited, Ind. Eng. Chem. Fundam. 18 (3), 199-208. 1092 doi:10.1021/i160071a001 
Machac, I., J. Cakl, J. Comiti, and N. E. Sabiri (1998), Flow of non-Newtonian fluids through

1095

1096

1097

1098

1099

1100

1101

1102

1103

1104

1105

1106

1107

1108

1109

1110

1111

1112

1113

1114

1115

1116

1117

1118

fixed beds of particles : Comparison of two models, Chemical Engineering and Processing, 37, 169-176.

Macosko CW (1994), Rheology: principles, measurements and applications, Wiley-VCH

Malvault, G. (2013), Détermination expérimentale de la distribution de taille de pores d'un milieu poreux par l'injection d'un fluide à seuil ou par analyse fréquentielle, $\mathrm{PhD}$ thesis, Arts et Métiers ParisTech.

Mei, C.C., and J. -L. Auriault (1991), The effect of weak inertia on flow through a porous medium, J. Fluid Mech., 222, 647-663. doi:10.1017/S0022112091001258

Miskimins, J. L., H. D. Lopez-Hernandez, and R. D. Barree (2005), Non-Darcy flow in hydraulic fractures: does it really matter?, SPE 96389 annual technical conference and exhibition, Dallas, 9-12.

Mongruel, A., and M. Cloitre (2003), Axisymmetric orifice flow for measuring the elongational viscosity of semi-rigid polymer solutions, J. Non-Newton. Fluid Mech., 110, 2743.

Müller, AJ, and A. E. Sáez, (1999). The rheology of polymer solutions in porous media, In: Nguyen TQ, Kausch HH (eds) Flexible polymer chain dynamics in elongational flow: theory and experiment, Springer, Heidelberg, pp 335-393. 
Nash, S., and D. A. S. Rees (2017), The Effect of Microstructure on Models for the Flow of a

Bingham Fluid in Porous Media: One-Dimensional Flows, Transport in Porous Media (accepted manuscript). doi:10.1007/s11242-016-0813-9

1122

1123

Neasham, J.W. (1977), The morphology of dispersed clay in sandstone reservoirs and its 1124 effects on sandstone shaliness, pore space and fluid flow properties, Paper SPE 6858 1125 presented at the 1977 SPE annual technical conference and exhibition, Denver. doi:10.2118/6858-MS

1127

1128

Nguyen, T.Q., and H. H. Kausch (1999), Flexible Polymer Chains in Elongational Flow: Theory and Experiement, Springer, Berlin.

1130

1131 permeability reservoirs symposium, Denver. doi:10.2118/16400-MS

1134

1135

1136

Neuzil, C. E., and J. V. Tracy (1981), Flow through fractures, Water Resour. Res., 17(1), 191-199, doi:10.1029/WR017i001p00191.

Nowamooz, A., G. Radilla, M. Fourar, and B. Berkowitz (2013), Non-Fickian Transport in 1139 doi:10.1007/s11242-013-0165-7 
Oukhlef, A., S. Champmartin, and A. Ambari (2014), Yield stress fluids method to determine the pore size distribution of a porous medium, Journal of Non-Newtonian Fluid Mechanics, 204, 87-93.

Palaniraj, A., and V. Jayaraman (2011), Production, recovery and applications of xanthan gum by Xanthomonas campestris, J. Food Eng., 106, 1-12.

Panfilov, M., and M. Fourar (2006), Physical splitting of nonlinear effects in high-velocity stable flow through porous media, Adv. Water Resour., 29, $30-41$.

Pascal, H. (1983), Nonsteady flow of non-Newtonian fluids through a porous medium, Int. J. Eng. Sci., 21, $199-210$.

Perkowska M, G. Mishuris, and M. Wrobel (2016), Universal hydrofracturing algorithm for shear-thinning fluids: particle velocity based simulation, Comput Geotech, 71, 310-37.

Perrin, C. L., P. M. J. Tardy, K. S. Sorbie, and J. C. Crawshaw (2006). Experimental and modeling study of Newtonian and non-Newtonian fluid flow in pore network micromodels., J. Colloid Interface Sci., 295(2), 542-550. doi:10.1016/j.jcis.2005.09.012.

Pipe, C. J., T. S. Majmudar, T. S., and G. H. McKinley (2008), High shear rate viscometry, Rheol Acta, 47, 621-642, doi:10.1007/s00397-008-0268-1 
Radilla, G., A. Nowamooz, and M. Fourar (2013), Modeling Non-Darcian Single- and TwoPhase Flow in Transparent Replicas of Rough-Walled Rock Fractures, Transp Porous Med, 98, 401-426. doi:10.1007/s11242-013-0150-1

Rao, P.T., and R. P. Chhabra (1993). Viscous non-Newtonian flow in packed beads: effects of column walls and particle size distribution, Powder Technology, 77, 171-176.

Rasoloarijaona, M., and J. L. Auriault (1994). Nonlinear seepage flow through a rigid porous medium, Eur. J. Mech. B/Fluids, 13 (2), 177-195.

Rocha, R.P.A., and M. E. Cruz (2010), Calculation of the permeability and apparent permeability of three-dimensional porous media, Transp. Porous Media, 83 (2), 349-373. doi:10.1007/s11242-009-9445-7

Rodríguez, S., C. Romero, M. L. Sargenti, A. J. Müller, A. E. Sáez, and J. A. Odell (1993). Flow of polymer solutions through porous media, J Non-Newton Fluid Mech, 49, 63-85.

Rodríguez de Castro, A. (2014), Flow experiments of yield stress fluids in porous media as a new porosimetry method, $\mathrm{PhD}$ thesis, Arts et Métiers ParisTech. https://pastel.archivesouvertes.fr/file/index/docid/1068908/filename/RODRIGUEZ_-_DE_-_CASTRO.pdf 
Rodríguez de Castro, A., A. Omari, A. Ahmadi-Sénichault, and D. Bruneau (2014), Toward a

1187

1188

1189

1190

1191

1192

1193

1194

1195

1196

1197

1198

1199

1200

1201

1202

1203

1204

1205

1206

1207

Rodríguez de Castro, A., A. Omari, A. Ahmadi-Sénichault, S. Savin, and L.-F Madariaga, (2016a). Characterizing Porous Media with the Yield Stress Fluids Porosimetry Method, Transport in Porous Media, 114(1), 213 - 233. doi:10.1007/s11242-016-0734-7.

Rodríguez de Castro, A., M. Oostrom, and N. Shokri (2016b). Effects of shear-thinning fluids on residual oil formation in microfluidic pore networks, Journal of Colloid and Interface Science, 472, 34 - 43. doi:10.1016/j.jcis.2016.03.027.

Rodríguez de Castro, A., and G. Radilla (2016a), Non-Darcian flow experiments of shearthinning fluids through rough-walled rock fractures, Water Resour. Res., 52(11), 9020 9035. doi:10.1002/2016WR019406.

Rodríguez de Castro, A., and G. Radilla (2016b), Non-Darcian flow of shear-thinning fluids through packed beads: experiments and predictions using Forchheimer's law and Ergun's equation, Advances in Water Resources, 100, 35 - 47, doi: 10.1016/j.advwatres.2016.12.009

Roustaei, A., T. Chevalier, L. Talon, and I. A. Frigaard (2016), Non-Darcy effects in fracture flows of a yield stress fluid, J. Fluid Mech., 805, 222-261. doi: 10.1017/jfm.2016.491 
1209 Scheidegger, A.E. (1960), The physics of flow through porous media, Macmillan, New York.

1210

1211 Sabiri, N.-E., and J. Comiti (1995), Pressure drop in non-Newtonian purely viscous fluid flow 1212 through porous media, Chemical Engineering Science, 50, 1193-1201.

1214 Schneebeli, G. (1955), Expériences sur la limite de validité de la loi de Darcy et l'apparition 1215 de la turbulence dans un écoulement de filtration, La Houille Blanche, 2, 141-149. 1216 doi:10.1051/lhb/1955030

1217

1218 Seright, R.S., T. Fan, K. Wavrik, and R. de Carvalho Balaban (2011), New Insights Into 1219 Polymer Rheology in Porous Media, SPE Journal, 16, doi:10.2118/129200-PA.

1220

1221

Sheng, J.J. (2011): Modern Chemical Enhanced Oil Recovery, Theory and Practice, GPG.

1222 Elsevier, Boston.

1223

1224 Silliman, V. (1989). An interpretation of the difference between aperture estimates derived 1225 from hydraulic and tracer tests in a single fracture, Water Resources Research, 25 (10), $12262275-2283$. 
Silva, J. A. K., M. Smith, J. Munakata-Marr, and J. E. McCray (2012), The Effect of System Variables on In situ Sweep-Efficiency Improvement via Viscosity Modification, J. Contam. Hydrol. 136, $117-130$.

Smit, G. J. F., and J. P. du Plessis (1997). Pressure drop prediction of power law fluid through granular media, J. Non-Newtonian Fluid Mech., 72, 319-323.

Sochi, T., and M. J. Blunt (2008), Pore-Scale network modeling of Ellis and HerschelBulkley fluids, J. Petr. Sci. Eng., 60, $105-124$.

Song, K.-W., Y. -S. Kim, and G. S. Chang (2006): Rheology of concentrated xanthan gum solutions: steady shear flow behavior, Fibers Polym., 7(2), 129-138

Song, Y.-Q. (2007). Novel NMR techniques for porous media research. Cement and Concrete Research, 37, 325-328.

Sorbie, K. S., P. J. Clifford, and R. W. Jones (1989), The Rheology of Pseudoplastic Fluids in Porous Media Using Network Modeling, Journal of Colloid and Interface Science, 130, 508534.

Sorbie, K.S. (1991a), Polymer-Improved Oil Recovery. Blackie and Son Ltd, Glasgow. 
Sorbie, K.S. (1991b), Rheological and transport effects in the flow of low-concentration xanthan solution through porous media, J. Colloid lnterface Sci., 145(I), 74-89.

Talon, L., H. Auradou, and A. Hansen (2014). Effective rheology of Bingham fluids in a rough channel, Frontiers in Phyisics, 2:24. doi:10.3389/fphy.2014.00024

Tiu, C., J. Z. Q. Zhou, G. Nicolae, T. N. Fang, and R. P. Chhabra (1997). Flow of Viscoelastic Polymer Solutions in Mixed Beds of Particles, The Canadian Journal of Chemical Engineering, 75, 843-850.

Tosco, T., D. L. Marchisio, F. Lince, and R. Sethi (2013), Extension of the DarcyForchheimer Law for Shear-Thinning Fluids and Validation via Pore-Scale Flow Simulations, Transp Porous Med, 96, 1-20. doi:10.1007/s11242-012-0070-5

Truex, M., V. R. Vermeul, D. T. Adamson, M. Oostrom, L. Zhong, R. D. Mackley, B. G. Fritz, J. A. Horner, T. C. Johnson, J. N. Thomle, D. R Newcomer, C. D. Johnson, M. Rysz, T. W Wietsma, and C. J. Newell (2015), Field Test of Enhanced Remedial Amendment Delivery Using a Shear-Thinning Fluid, Groundwater Monitoring and Remediation, 35, 3445. doi: $10.1111 / \mathrm{gwmr} .12101$.

Tsang, Y. W., and C. F. Tsang (1987), Channel model of flow through fractured media, Water Resour. Res., 23(3), 467-479, doi:10.1029/WR023i003p00467. 
Wadhai, V.S., and A. N. Dixit (2011), Production of Xanthan gum by Xanthomonas campestris and comparative study of Xanthomonas campestris isolates for the selection of potential Xanthan producer, Indian Streams Res. J., 1, 1-4.

Wang, M., C. Yi-Feng, M. Guo-Wei, Z. Jia-Qing, and Z. Chuang-Bing (2016), Influence of surface roughness on nonlinear flow behaviors in 3D self-affine rough fractures: Lattice Boltzmann simulations, Advances in Water Resources, 96, 373 - 388.

Wever, D. A. Z., F. Picchioni, and A. A. Broekhuis (2011), Polymers for enhanced oil recovery: A paradigm for structure-property relationship in aqueous solution, Progress in Polymer Science, 36 (11), 1558-1628.

Withcomb, P.J., and C. W. Macosko (1978): Rheology of xanthan gum., J. Rheol., 22, 493.

Witherspoon, P.A., J. S. Y. Wang, K. Iwai, and J. E. Gale (1980), Validity of cubic law for fluid flow in a deformable rock fracture, Water Resour. Res., 16 (6), 1016-1024. doi:10.1029/WR016i006p01016

Woudberg, S., J. P. Du Plessis, and G. J. F. Smit (2006). Non-Newtonian purely viscous flow through isotropic granular porous media, Chemical Engineering Science, 61, 4299-4308. 
Xin, J., X. Zheng, J. Han, H. Shao, and O. Kolditz (2015). Remediation of trichloroethylene

1295

1296

1297

1298

1299

1300

1301

1302

1303

1304

1305

1306

1307

1308

1309

1310

1311

1312

1313 by xanthan gum-coated microscale zero valent iron (XG-mZVI) in groundwater: effects of geochemical constituents, Chem. Eng. J., 271, $164-172$.

Xiong, X., B. Li, Y. Jiang, T. Koyama, and C. Zhang (2011), Experimental and numerical study of the geometrical and hydraulic characteristics of a single rock fracture during shear, Int. J. Rock Mech. Min. Sci., 48(8), 1292-1302, doi:10.1016/j.ijrmms.2011.09.009.

Yazdchi, K., and S. Luding (2012), Towards unified drag laws for inertial flow through fibrous materials, Chemical Engineering Journal, 207 - 208, 35-48

Zhang, L., H. Sun, B. Han, L. Peng, F. Ning, G. Jiang and V. F. Chehotkin (2016), Effect of shearing actions on the rheological properties and mesostructures of CMC, PVP and CMC + PVP aqueous solutions as simple water-based drilling fluids for gas hydrate drilling, Journal of Unconventional Oil and Gas Resources, 14, 86 - 98.

Zhong, L., M. Oostrom, T. W. Wietsma, and M. A. Covert (2008), Enhanced Remedial Amendment Delivery through Fluid Viscosity Modifications: Experiments and numerical simulations, Journal of Contaminant Hydrology, $101,29 \quad-\quad 41$. doi:10.1016/j.jconhyd.2008.07.007 
Zimmerman, R. W., S. Kumar, and G. S. Bodvarsson (1991), Lubrication Theory Analysis of 1316 the Permeability of Rough-walled Fractures, Int. J. Rock Mech. Min. ScL \& Geomech. dbstr., $1317 \quad 28(4), 325-331$

1318

1319 Zimmerman, R. W., and I. W. Yeo (2000), Fluid flow in rock fractures: From the Navier1320 Stokes equations to the cubic law. In: Faybishenko, B., Witherspoon, P.A., Benton, S.M. 1321 (eds.) Dynamics of Fluids in Fractured Rock, Geophys. Monogr., 122, 213-224, AGU, 1322 Washington, DC.

1323

1324 Zimmerman, R. W., A. H. Al-Yaarubi, C. C. Pain, and C. A. Grattoni (2004), Non-linear 1325 regimes of fluid flow in rock fractures, Int. J. Rock Mech. Min. Sci. 41, 163-169. doi:10.1016/j.ijrmms.2004.03.036 